\title{
Spatio-Temporal Analysis on Urban Traffic Accidents: A Case Study of Tehran City, Iran
}

\author{
Niloofar Haji Mirza Aghasi \\ Graduate School of Life and Environmental Science, Division of Spatial Information Science, The University of Tsukuba, \\ Tsukuba, Ibaraki", Japan \\ Email:nia922@yahoo.com
}

How to cite this paper: Aghasi, N.H.M. (2018) Spatio-Temporal Analysis on Urban Traffic Accidents: A Case Study of Tehran City, Iran. Journal of Geographic Information System, 10, 603-642.

https://doi.org/10.4236/jgis.2018.105032

Received: January 25, 2018

Accepted: October 26, 2018

Published: October 29, 2018

Copyright $\odot 2018$ by authors and Scientific Research Publishing Inc. This work is licensed under the Creative Commons Attribution International License (CC BY 4.0).

http://creativecommons.org/licenses/by/4.0/ (c) (i) Open Access

\begin{abstract}
Urban Traffic Accidents (UTAs) may be seen as discrete events, localized in space and time. UTAs rates all over the world show great disparity, especially between developed and developing countries. Today, the most negative results of urban transportation are UTAs with many side effects such as injuries and loss of lives. UTAs lead to injury, death, disability and pain, loss of productivity, grief, social and mental problems. Proper and deep study and planning can enhance transport and driving safety and reduce number and severity of accidents. Traffic safety crises, death, damage and costs resulting from road UTAs are some of the most important public health and police organization challenges. In particular, UTA's victims are often people who are aged 15 - 44 years old in Iran, and UTAs are the second cause of death after heart disease in Tehran. UTAs' statistics in Tehran reveal a serious problem with significant fatality and injury rate. This study aims to identify the spatial pattern of UTAs in the city of Tehran in order to find the causes and consequences as well as the temporal and spatial or spatio-temporal variation of accidents. The relationship between the space and time of daily activities that generate urban daily trips and UTA, is examined in Tehran city for 2010 to 2011. The analysis is based on different primary and secondary data sources, which include locations of accidents and different attributes such as date, reason, kind, etc. Based on the data analysis, the study also attempted to show some light on the major causes, factors and types of accidents in order to identify the problem and suggest appropriate suggestions which reduce UTAs. As this study considered different factors of UTA, urban environment, land use, population, human activities and culture point considered as the most important pillars of this study. In order to understand knowledge, culture and attitudes of drivers towards traffic regulations, questionnaires were distributed to 1500 drivers in the study area to gather data about the drivers' knowledge, beliefs, attitudes, and behaviors and 1177 of them returned. The results express that culture and knowledge of drivers have direct effects on
\end{abstract}


localizing accidents. Furthermore, the concentration of educational, commercial and cultural activities that make up a large number of urban trips and urban dynamics, road usage, and time are among the main considerations of this study. The relationships between population, land use and dynamic patterns of city which constitute the urban structure, are used to establish a link between UTA and the urban structure of Tehran city. Time is considered as a crucial variable in this study that leads people to different kinds of locations and risk. Land use data and population data are combined with the accidents data using GIS techniques to generate relevant inputs for analysis. Concerning the methodology, cluster analysis techniques are developed to analyze the association between UTA numbers and land use categories and per 1000 residents of Tehran city. The techniques are developed to investigate the temporal variation of UTA over the time periods segmented into different zones. The results show that the suburban zone with industrial land use types and more highways are associated with more fatal and injured accidents. In comparison, the CBD zone is the safest not only in regarding the number of accidents but also in severity of accidents. Traffic limitation boundaries, wide pedestrians walking area and increased police checks, make this area safer despite the higher population density and daily activities. It was observed that the UTA spatial pattern changes dramatically in different zones and hours, especially during RH. Increased accidents but of lower severity happen in Tehran during the RH day when there is traffic congestion on transportation networks or roads and crowding on public transport is at its highest. Normally, this happens twice in a day, first in the morning and second in the afternoon-evening, the times during which the most people commute. It was observed that land use category, urban structure and population density make different rush hours in the city, therefore, different zones have different RHs. Referring to population movement or urban dynamic and urban structure, main roads types in urban and suburban zones become congested in different hours due to a large number of people activities. Thus, these different RHs in different zones result in different spatial patterns of accidents within the city. During Thursdays, the schools and administrative offices follow a half-day schedule and many people engage in recreation such as shopping and other extra activities. This causes a different type's RHs in Thursdays as it takes some load from the evening $\mathrm{RH}$, and thus makes the morning $\mathrm{RH}$ the most intense time of the day. In addition, this study provided an explanation on the relationship between urban structures, creating UTA in Tehran. It was discovered that the locational pattern of the various land uses in the urban area is a reflection of socio-economic and ecological factors. Furthermore, the spatial analysis and temporal analysis of relative accidents risks point out the risky segments for different zones of the city and different land uses depending on the season, month, day and time.

\section{Keywords}

Geographic Information System (GIS), Kernel Density Estimation, Land Use Rush Hour, Urban Structure, UTAs 


\section{Introduction}

\subsection{Background and Problem Statement}

Fatalities and injuries resulting from UTAs are recognized as a major problem in societies across the world as urban dynamics increase the development of transportation networks and demand for motor vehicles. Over 16,000 Iranians lost their lives in UTAs in 2014. According to the recent reports of the World Health Organization [1], one of the major problems in the world has been UTAs which result in approximately 1.24 million deaths and tens of millions of injuries and disabilities every year. As a developing country, associated with growing technology and an increasing population in Tehran, the number of vehicles in traffic is increasing day by day. The growth of Tehran City and high rate of migration have led to an increase in the need for mobility and a consequent increase in the usage of any type of vehicles occupying the urban transportation infrastructure. As a result, traffic exposure and thus, the number of UTAs, are growing. However, the normalized rates of UTAs per 1000 population or the total number of vehicles still show very high fatality and injury rates in Tehran, which signal a significant traffic safety problem that should be addressed and analyzed to find contributing factors. According to the statistics, UTAs are the second reason for death in Tehran City [2]. The severe situation of UTAs and the rate of fatalities and injuries caused by UTAs in Tehran and other cities are a critical problem that needs to be dealt with urgently. It is necessary to examine the main causes of UTAs to find a solution. Some studies have been conducted for analyzing UTAs around the world. However, to develop effective countermeasures, especially in the case of Tehran, UTAs should be analyzed spatially in a Geographic Information Systems (GIS) environment that identifies their occurrence time, type, results, and coordinates. Ultimately, identifying high probability UTA areas is an important issue of traffic safety programs, because precautions and infrastructure assessments can be prioritized at these locations to create more efficient resource allocations. Such areas are called "black spots" or "hotspots" in the UTA literature [3] and generally they are detected by clearly defined methods. On the other hand, identification of such problematic areas in the urban structure and the accident's location is more complicated; this is loosely defined as a "hotspot" in the urban traffic safety literature, mainly because the traffic conditions, UTA mechanisms, and preventive measures differ greatly in urban traffic networks. Moreover, the "hotspot" definition can vary according to the focus of the analysis.

Secondly, any solution or improvement option may require designs and precautions beyond the changes made to the transportation network's qualities, knowledge of drivers, driving rules, and optimization of culture. Based on the scope of a hotspot detection analysis, the criteria for detecting a minimum number of UTAs, UTA type, occurrence time, occurrence area, etc. can be changed over time. UTAs occurring in proximity spatially may be due to higher 
levels of daily urban trips and urban dynamics, conflicting movements after detection, rule breaking, etc. It is also important to analyze the distribution of hotspots in cities, which are not always distributed uniformly over space or time. Urban built environments and transportation networks have always had an active interaction that has both direct spatial and temporal dimensions. Land use patterns affect the travel behavior in both trip generation and trip distribution, so that more trips are generated and distributed in some regions, such as the central business district (CBD), than others. Time is one of the critical parameters in UTA analysis, as there is a strong relationship between the urban structure (US) and number of UTAs.

Higher numbers of pedestrians are expected in some areas, such as the CBD, or near shopping centers, schools and universities, administration offices, and health facilities in urban zones. For this reason, hotspots should be evaluated together with urban structures or urban built environment characteristics, and temporality. In the absence of traffic volume data for Tehran City, other exposure measures, such as geographical area and transportation network characteristics, including road types, were used in this study. Analyzing UTA hotspots together with time and US or urban zones is important to drawing conclusions about which areas have priority in terms of infrastructural regulations and the application of new traffic limitations and restrictions. As the pattern of UTAs differs temporally and by zones, the relationship between them should be analyzed and evaluated.

\subsection{Research Objectives}

The main objective of this study is doing empirical study that would lead to the detection and understanding of the distribution of UTAs and identifying UTA hotspots in the US using GIS coupled with spatial analysis methods. The scheme includes the following: identifying the pattern of UTAs through the spatial structure of the city, which is three zones based on my methodology; identifying accident hotspots based on land use and population using clustering methods; and identifying the distribution and pattern of hotspots over different city zones of Tehran by time (which is a crucial factor), and the distribution of hotspots over different time periods (rush hours and regular hours).

The first step for identifying the hotspots is through the use of the clustering method. When finding hotspots, UTAs can be used with or without groupings by type and time. To detect hotspots, a clustering method is used with the ratio and rate of UTAs determined according to the great differences between them in terms of number of UTAs. Hotspot severity is defined as the number of UTAs found within each cluster, which also takes into consideration the analysis of hotspot distances to the nearest land use, and the population polygon. This study also uses visualization techniques to show hotspots and their severity values. Finally, the relation between hotspots, the built environment and land use category are checked to determine the high-risky land use. 


\subsection{Geographical Setting of the Study Area}

The study area of this research is Tehran City (Figure 1), which is located in the Tehran Metropolitan Area and has a daytime population of approximately 12,000,000 according to the Presidency of the IRI Plan and Budget Organization, Statistical Center of Tehran report [4]. Tehran City is the most important and most dynamic city in Iran as it is the main center of all governmental and public offices, major universities, the main medical port of Iran, the main center of administration offices, and so on. As the capital of Iran, Tehran is one of the most important cities not only in Iran, but also in the Middle East and is the center of many national and international organizations; it has experienced a rapid growth in population and is surrounded by different suburban cities, such as Karaj. Karaj is the most important countryside city of Tehran with a population $1,614,624$ [5] and is within an hour's distance from Tehran by road, the metro, and other public transportation as well. Karaj is one of the Iran's metropolises and the short distance between this metropolis and Tehran via various transportation modes, causes 14,240 daily trips [6] for different purposes, such as health, education, work, and other activities. There are many residents of Karaj who work or study in Tehran because of cheaper land prices in Karaj. They are involved in round trips from Karaj to Tehran every day but especially on weekdays. In addition, the most important and biggest stadium of Iran, the Azadi Stadium, is located on the Tehran-Karaj connecting highway. Sporting events at the Azadi Stadium, weekends and holidays, and some cultural and religious activities create different patterns of traffic and UTAs within Tehran City and the commuting roads between Tehran and Karaj City.

As the capital and most important city of Iran with respect to politics, science, health, education, etc., Tehran is also the most populous city even if it is not the largest. Its population varies greatly between night and day; the daytime population is close to 12 million while the night-time population is over 8.5 million [5]. The span of Tehran City is 730 square kilometers, and the important role of the city gives it an international sister city relationship to some other great cities and metropolises, such as Seoul, Moscow, Peking, Istanbul, etc. Before the Islamic revolution these sister cities included Los Angeles and Seoul. The altitude of Tehran City is 2000 meters at the highest northern parts, and 1050 meters above sea level to the south (the municipality of Tehran). Tehran is surrounded by the Alborz Mountains and comprises 22 districts with their own administrative centers, 123 areas, and 374 regions. Tehran borders the Shemiranatin the north, the Damavand in the east, the Eslamshahr, Pakdasht, and Rey counties to the south, and finally, the Karaj and Shahriar country sides to the west.

\section{Materials and Methods}

\subsection{Data Collection}

This study, like others, uses various databases to examine UTAs. In this study, I used four datasets to develop a GIS of UTAs in Tehran. The methods, design, 


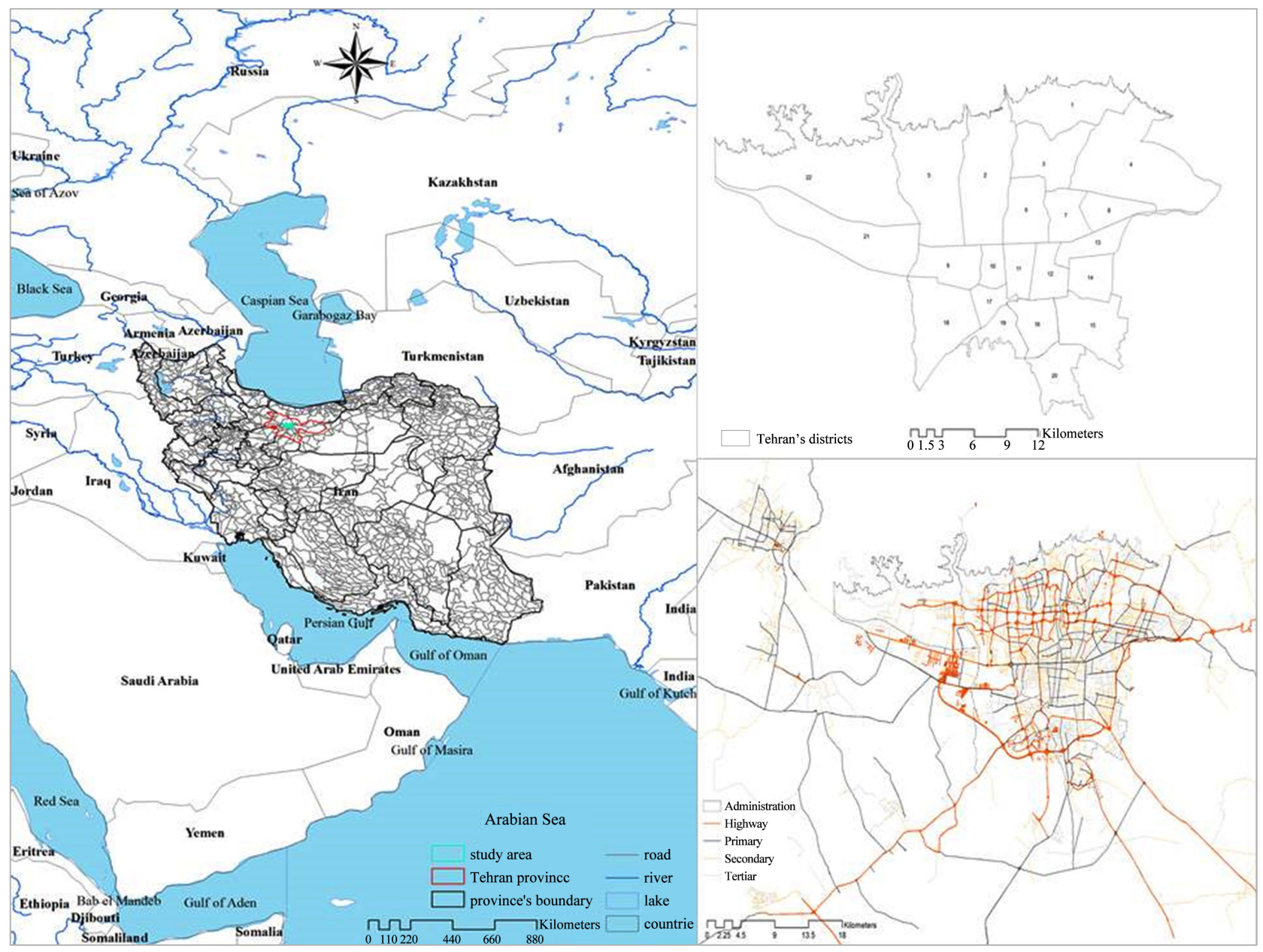

Figure 1. Study area.

and visual representations of the UTAs are widely accepted within the literature on the topic, and are useful and relevant. ArcGIS is used to create the GIS and contains the datasets of the UTAs in Tehran. The first important main dataset (the Tehran UTA database) was received in January 2013. The data was obtained through the Tehran Police Department. Its format is Microsoft Excel (2007) and it contains over 64,000 cases of UTAs within a year (2011). The attributes of each UTA in the data include location, weather conditions, brightness, severity of UTA (damage, injury, fatal), exact date, day of the week, and time of day. The location is defined by address and name. Each UTA is unique in containing the attributes listed. The UTA will be placed where it occurred in time and space on the transportation network of Tehran.

The second data base is the Tehran Transportation Network (TTN) data base that was received in October 2012; the data was obtained through the Tehran Transportation Organization. The format was not usable in GIS format because of some language issues and some missing information. However, the database was developed and made ready by the author.

The third database concerns land use. Until very recently, serious and efficient 
attempts in national land use planning in Tehran had never been undertaken. Thus, the author created a land use database shape file using a geo-digitizing technique. The main database is a by-product of a 2011 Google map and a map of Tehran. The experimental study uses highly detailed Google satellite images and Google Earth, which allow the identifications of dominant land use category and urban facilities in the land use database.

The forth database, (population), was obtained from the PIRIMPO and Budget Organization, Statistical Center of Iran and Landsat data for 2011.

In addition, in order to gather data about the drivers' knowledge, beliefs, attitude, and behavior, 1500 questionnaires were handed out in the study area and 1135 were returned. Questionnaire forms were prepared to obtain original new data and a better understanding of drivers' self-reporting. The questionnaire was distributed in Tehran zones among random Tehran drivers between June 15, 2015 and July 02, 2015. Furthermore, to enhance originality and obtain additional and unique new data, fieldwork was undertaken in the study area in June, 2015. The methodology of this field work involved use of a traffic conflict technique.

\subsection{Methodology}

To understand the hotspots or black spots of the UTAs in Tehran City, the kernel density method was used as an appropriate hotspot analytic tool to provide the best black location of UTAs in the city. Furthermore, in I used cluster analysis and the risky area divided into four categories based on the number of accidents in land use analysis, and three categories for population analysis [7]. The method has been used because the interpretation of land use, population density, and UTAs are required given the research scope; using this method it was possible to classify UTAs by land use and population into the same groups. Different databases and shapefile were also used in this study but the central and most important data, which are the point data and different attributes such as date, time, reason, and kind of UTAs, are from the UTA database. The attributes are varied and include the real time of the UTAs, date of UTAs, kind of UTAs (dead, injured, or losses experienced), type of UTA (car by car, car by motorcycle, car by pedestrian), and the reason for the UTA (lack of attention, lack of priority, high speed, etc.).

For better understanding of the relationship between UTAs and urban structure, Tehran City is divided into three different zones in this study, and these three different zones are analyzed separately (Figure 2). These three zones were the CBD, the urban zone, and the suburban areas of Tehran. This selection was made by the author and by considering Tehran's master plan, dominant land use category, historical geography of the city, and personal knowledge and experience. Although the CBD zone, urban, and suburban zones are adjacent parts of the network, it is important to consider these separately as the three zones have different environment characteristics owing to their different structure. Tehran's 


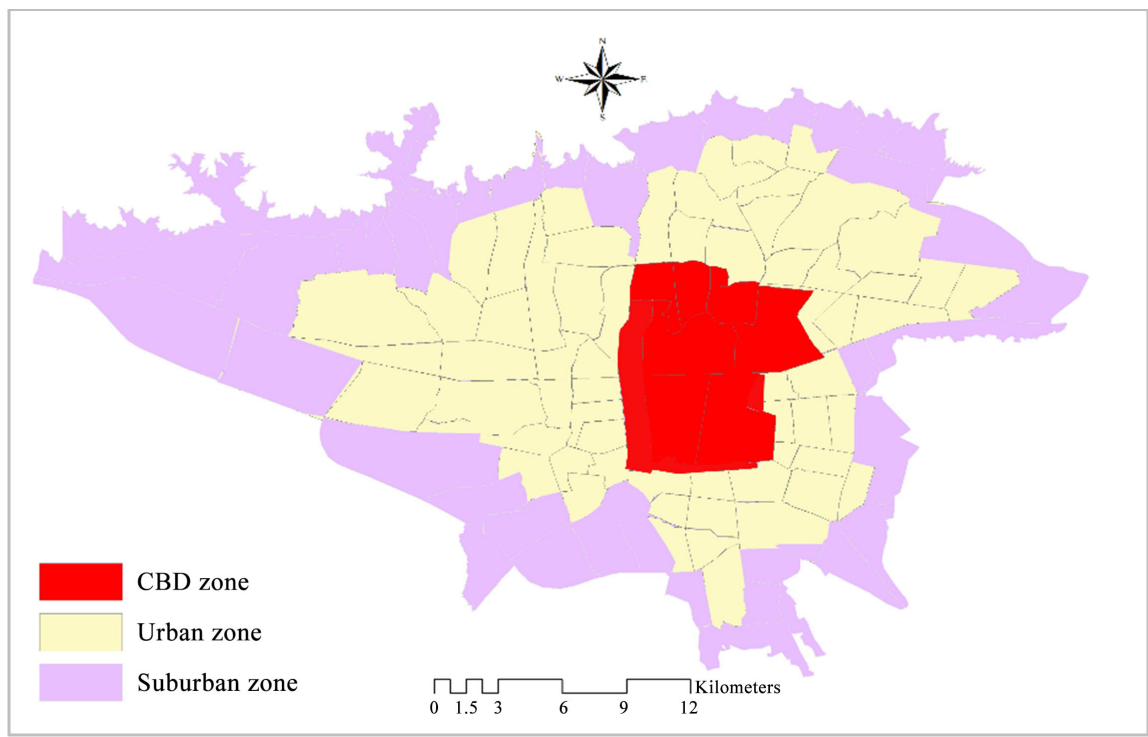

Figure 2. Tehran's zone classification.

CBD is located in east center of the city and include main streets of the Grand Bazaar and consists of commercial and administrative land use categories and a few residential areas. Tehran's urban zone was decided upon as it has higher population density, a high rate of road networks, and greater facilities and buildings density. Tehran's urban zone is in a different land use category and is the main zone of Tehran's residential areas. The suburban zone is characterized by less population density, and consists of an industrial and transportation land use category with more highways and fewer residential roads.

\section{Spatial Distribution of Accidents in Tehran}

The pattern and location of where the UTAs are located in the transportation network within Tehran City can provide a detailed and meaningful understanding of tendencies. To gain such information and analytic results from the UTAs in space and time, the cluster analysis in the ArcGIS software has been used. The spatial arrangement of the points' features is an important characteristic of a spatial pattern because the location of point features and the relationships among them have a significant effect on the underlying process that generates a distribution. The databases of understanding the spatial arrangement of the UTAs are substantial not only in Tehran, but also in all other big cities like Tehran, which trace the pattern of occurrences of the UTAs. The spatial distribution of UTAs in Tehran City clearly shows that there were some locations with multiple occurrences of UTAs and that most of these points are located in highways and freeways first, and then in residential roads. Moreover, spatial association in point pattern analysis evaluates relationships between points and features and other sets of variables, so it can be useful to relate the location of Tehran UTAs to the other features to examine the dispersion of point patterns against other factors. Spatial associations can be explored by overlaying a combination 
of points, lines, and polygon features. Identification of the spatial and temporal distribution patterns of UTAs, and identification of the associated risk factors thorough the US and socio-economic factors is the key to the best decision-making; identifying these patterns can increase the quality of human safety, future planning and understanding which areas are priorities for implementing safety programs. After obtaining the UTA data from the Tehran Police, the first step was to preprocess the database by refine it for incorrect entries or those lacking latitude or longitude attributes. The data were then arranged based on temporal characteristics. UTAs generally occurred during the summer months in all three zones. One of the most differences between seasons is weather characteristics. While it is cold and snowy in the winter, the heat from the sun in the summer causes high temperatures. These differences affect drivers' behaviors and safety. As UTA numbers increase in hot weather, the majority of all accidents occurred in summer partly as a result of personal exposure and use of private cars instead of public transportation or walking even a short distance; people going on vacation as schools are closed down also contributes to an increase in car accidents but this decreases in spring and winter. In spring, the number of UTAs decreases in urban areas, but increases on suburban roads. This is because of the New Year's holiday (Norooz) and the increase of trips from inside the city to outside; in the winter season it is because road users drive more carefully. The finding might be attributed to culture.

Also, the UTA rate is low in the winter, which suggests that seasonal weather conditions have less effect on the quantity and quality of accidents. From the results of this study, it is very clear that seasons have less effect on UTAs but some months affect accident rates because of the organization of events that occur during them. As with Mehr, which is the first month of the academic calendar, or Farvardin and Esfand, which are first and the last month of the year, respectively. In all of these months, culture and costumes are important. For example, in September (Mehr), on the first day of the academic year, most students go to school with their parents in private cars rather than using school or public transportation services. Many of these students have school transportation services or are old enough to use public transportation, but they go to school with their parents on the first day and in many cases throughout the first week. Likewise, in the second half of Esfand, which begins March 7, people prepare many things for the New Year, which begins on March $21^{\text {th }}$; by custom, they have to buy new clothes, special foods and, in some cases, new things for the home. This shopping culture makes the city more dynamic and increases mobility at several times. The $\mathrm{CBD}$ and the urban zone are at the center of these activities. At the New Year holidays, which continue through April $6^{\text {th }}-7^{\text {th }}$, the CBD and urban zone have the lowest mobility rate and there is little congestion on the transportation network. However, UTAs are relatively low in winter for the CBD and urban zone, and in the spring for the suburban zone, as spring is Iran's New Year (Norooz, Farvardin $1^{\text {th }}-13^{\text {th }}$ ) and all schools, universities, administration of- 
fices, and the business district are almost closed for more than two weeks. This means less urban mobility and less daytime population are caused by the season, and this equals fewer UTAs in Tehran. In addition, many Tehran residents travel out of the city during this month. Furthermore, there are more UTAs on weekdays in the summer months than in other months as schools and universities are closed in summer and students and their families have more time for leisure and entertainment. In addition, because of hot weather most prefer to use their own cars instead of public transportation or walking and these behaviors make city more dynamic and cause more accidents. The zones' UTAs also reveal the pattern of operating hours, activities, and dynamic hours showing higher UTA rate than in the morning, evening, or night.

UTA patterns vary by month and day as well. For all UTAs, September and July, Tuesdays and Wednesdays, are the black months and days. Comparatively, September, July, and October are the months with the highest number of UTAs in the city where they contribute $11.3 \%, 10.3 \%$, and $10.1 \%$, respectively, to the total UTAs. This could mainly be due to the effects of time in schools and universities. The occurrence of UTAs can vary within the 24 hours of a day across zones. Environmental factors like the availability of light, dominant land use category, the number of pedestrians, and closest facility type have a significant impact on the kind and type of UTA distribution within a day. From a spatial perspective, there are noticeable geographic variations in Tehran's UTAs by zone (Figure 3). The variation in the hours of a day exhibits a difference in UTA occurrences. In the records, 39.5\% of UTAs were observed during rush hour. Ironically, the time between midnight and $06: 00$ contributes only $7.8 \%$ of UTA records in the city during the study period. UTAs in Tehran City are frequently observed in the daytime rather than at night-time between 18:00 and 22:00. About $71.21 \%$ of all the UTAs recorded during the study period have been observed at the time mentioned. The other $28.79 \%$ of UTAs have been recorded at night between 22:00 pm and 06:00 am. This means, driving or traveling on the TTN between 12:00 and 18:00 is five times more dangerous than driving or traveling between 22:00 pm and 06:00 am. This phenomenon is evident mainly because of the urban dynamic as the movement, number of cars, and volume of pedestrians increases during the day and makes a big difference compared to the dynamic at night. Comparing the UTAs within zones shows that the intensity and incidence of UTAs are positively correlated with time and space. Something which accounts for these differences is the type of transportation network and land use category, in short-urban structure. Based on the results of hotspot analysis after residential land use, the three most risky areas are industrial land use with 5054 accidents and 147 hot clusters; commercial land use with 4069 accidents and 121 hot clusters; and administration land use with 2639 accidents and 120 hot clusters. In addition, the results revealed that different land use categories create different black hours and different reasons for accidents. The main reasons for accidents in the industry land use category are high 


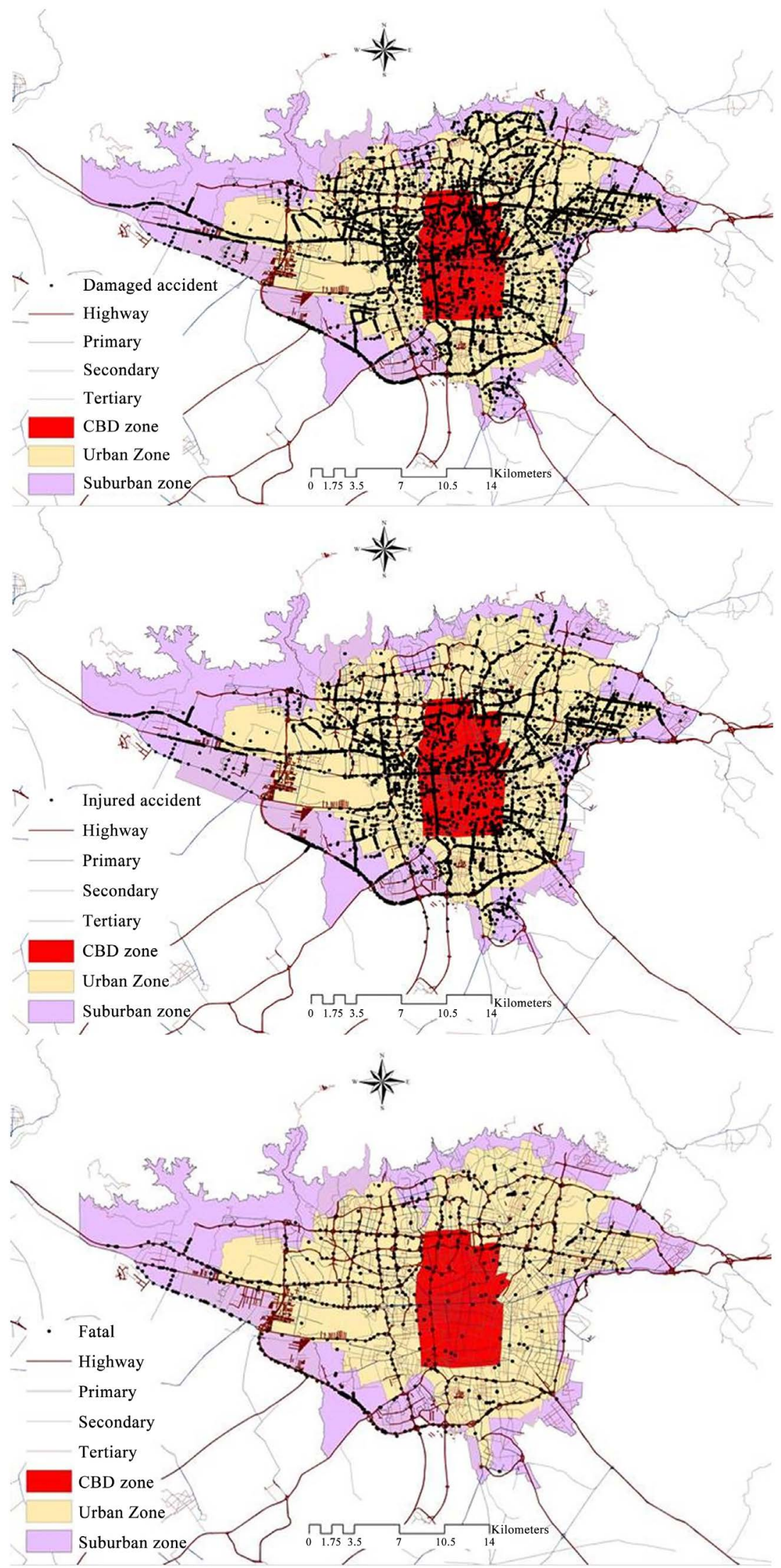

Figure 3. Spatial distribution of UTAs. 
speed, lack of attention, and sleepiness while in commercial and administration land use the reasons are lack of attention and pedestrian mistakes. The black time in the industrial area is 5:00-7:00 while it is 16:00-20:00 in the commercial area and 14:00-16:00 for the administration land use category.

My findings from this study revealed that land use category has an effect on the number and severity of accidents but has no impact on transportation mode, traffic volume, and urban daily trip rate.

\subsection{Temporal Distribution of UTAs}

The relationship between weather and UTA is not simple, but evident nonetheless. Seasons have less effect on accidents than other factors within cities. A study has been done based on temporal factors and outcomes showed that increase in UTA and accidents starts from June and include autumn months. It is concluded that this increasing caused by the fact that most residents travel from their locations to do entertainment in holiday area and that unfavorable weather conditions exist in autumn months [8]. The largest differences between seasons are weather characteristics and brightness (Table 1). While in winter it is cold and snowy, high temperature in dry Tehran in summer. These differences affect drivers' behavior and traffic safety. In Tehran city, UTA numbers increase in hot weather, partly as a result of road users deciding to take their own cars instead of walking or using public transportation, and decrease in winter, because road users drive more carefully and usually residents prefer to stay at home instead of move in the city.

Table 1. Monthly distribution of Tehran's UTAs (2011).

\begin{tabular}{ccc}
\hline Month & Number of Accidents & Percentage of Accident \\
\hline January & 3865 & $6 \%$ \\
February & 3543 & $5.5 \%$ \\
March & 4251 & $6.6 \%$ \\
April & 5797 & $9 \%$ \\
May & 4895 & $7.6 \%$ \\
June & 5540 & $8.6 \%$ \\
July & 6635 & $10.3 \%$ \\
August & 6313 & $9.8 \%$ \\
September & 7279 & $11.3 \%$ \\
October & 6506 & $10.1 \%$ \\
November & 5346 & $8.3 \%$ \\
December & 4573 & $7.1 \%$ \\
Total & 64,421 & $100 \%$ \\
\hline
\end{tabular}

Source: The Police Department of the I.R.I, Tehran. 
In respect of many researchers [9] that stated the number of accidents is higher in rainy and snowy days (autumn and winter), in Tehran city the share of accidents is higher in summer. $34 \%$ of accidents occurred in the summer, when the school and university classes are mostly closed or open only for some hours. The majority of all accidents occurred in summer and the lowest share is related to spring. In spring the number of UTA decrease in urban areas, but increase in suburban roads. This is because of the New Year Holiday (Norooz) and the increase of trips from inside of the city to outside. In this period of time the number of accidents outside of the city increases. However, in summer, the highest season in UTAs, increased temperatures, schools being closed down and people on the move towards vacation places contribute to an increase of UTAs.

The frequency of the UTAs by month increase in summer and autumn and decrease in winter within the Tehran City. As drivers do more attention in winter and less in summer because of high temperature. During the winter, people prefer to stay at home because of the weather condition and lack of sufficient urban transportation in rainy and snowy days, therefore population is inhibitory in the city and urban activities decrease.

Based on the temporal analysis of UTAs, $21 \%$ of UTAs occurred in spring, $34 \%$ in summer, $27 \%$ in autumn and $18 \%$ in winter.

Month of accidents is one the basis of attribute data that are collected for this study, therefore can observe the disparity among months in their total number of road UTAs. The relationship between UTA's and month is similar with seasons as months create different seasons. But some months have significant effects on UTAs. Such as September (Mehr) which is the first month of new educational year or Ramadan which change over the year. These difference characters of months and Ramadan as well, affect drivers' behavior and urban dynamic. UTA numbers decrease in winter, because road users drive carefully and most of the people who don't have official jobs and house wives prefer to stay at home, in addition employees and workers usually reduce work hours or take rest during snowy days. In addition, school closures because of snow are common in Iran and Tehran as well. Table 1 shows, the variation in the number of road UTAs during the months of a year. UTAs are fairly uniformly distributed by month ranging between $11.3 \%$ in September and 5.5\% in February, and accidents were relatively higher in the dry months of July, September and October than other months.

UTAs are analyzed based by daytime changes as these have a direct effect on urban movements and resident's daily pattern of activities.

The most significant number of UTAs in Tehran City are registered on Wednesdays (18.1\%), followed by Tuesdays (16.2\%), and the lowest number are on Fridays (11.3\%). It should be mentioned here that Saturdays and Sundays are weekdays and Fridays are weekends in Iran. The facts behind this weekly pattern can be attributed to urban dynamics and human mobility as well as traffic volume and pattern, which differ on weekdays and weekends; traffic volume and 
pattern is the key factor of connection between population and work places. Thursday is the final workday of the week and the population then takes longer trips to suburban zone with longer distance, for entertainment and relaxation in addition to workplace travel. Fridays has less urban movement and fewer daily urban trips are made as it is the only day off and all administration offices, schools, universities, and the Grand Bazaar are closed. Friday is the safest day of the week with the least number of UTAs, because of fewer business, employment, and educational trips being made. Thursday has the heaviest percentage of UTAs. As all schools, universities, government, and public positions are closed on Fridays, the lowest UTA occurrence (on this day) can be attributed to the lower number of urban trips. Most of the above-mentioned facilities close earlier on Thursdays than on other weekdays, but culturally and socially, people engage in other activities during the remaining time. Most shopping, weddings, parties and many other ceremonies and activities are held on Thursday nights. The weekly distribution of UTA severity is indicated in Table 2. The peak days for UTAs are Mondays and Thursdays.

Comparing the relationship between different hours of the day and the UTAs in Tehran, the incidence and intensity of UTAs correlate positively with RH and urban dynamics. UTAs are higher within Tehran City during the morning when offices, schools, and commercial centers open and then during the afternoon $\mathrm{RH}$ when offices, schools, and other governmental centers close and people are on the transportation network to return home. The pattern may be because of the intensity of trip production and its attractiveness for many different purposes, especially work or study. In addition, on Thursday government offices close early and employees usually work half a day or do not work at all, thus they have more time to go home for lunch and make more urban trips in the same day. Because the numbers driving at night are normally lower than in the daytime most UTAs in Tehran occur in daytime during the day hours. The hourly variation of Tehran's UTAs is shown in Figure 4. The table shows the coincidence of higher UTAs during the RH at morning, noon, afternoon, and evening.

To investigate a meaningful relation between numbers of UTAs in $\mathrm{RH}$, the ratio of accidents is calculated hour by hour. When the transportation networks are more crowded during the $\mathrm{RH}$, the rate of accidents is affected by lower speed and more accidents but they are less severe. The driving speed is the key factor which is dependent on traffic volume, road type and driver's attitude which expresses that relationship between UTA. Road type variations clearly show that in contrast to many cities in the world, Tehran's highways and expressways are not the most dependable for driving.

The urban traffic accident rate is higher during the morning when offices, schools, and commercial centers open and then during the afternoon peak hours when offices, schools, and other governmental centers close. This is because of the intensity of trip production for many different purposes, especially work and study. The figure demonstrates the coincidence of higher accidents during the $\mathrm{RH}$ in the morning, afternoon, and evening. 
Table 2. Daily distribution of Tehran's UTAs (2011).

\begin{tabular}{cccc}
\hline Day & Accident & Percentage \\
\hline Saturday & 9341 & $14.5 \%$ & $13.1 \%$ \\
Sunday & 8439 & $14.7 \%$ & $16.2 \%$ \\
Monday & 9469 & $18.1 \%$ \\
Tuesday & 10,436 & $12.1 \%$ \\
Wednesday & 11,660 & $11.3 \%$ \\
Thursday & 7797 & 7279 & $100 \%$ \\
Friday & 64,421 & \\
Total & & \\
\hline
\end{tabular}

Source: The Police Department of the I.R.I, Tehran.

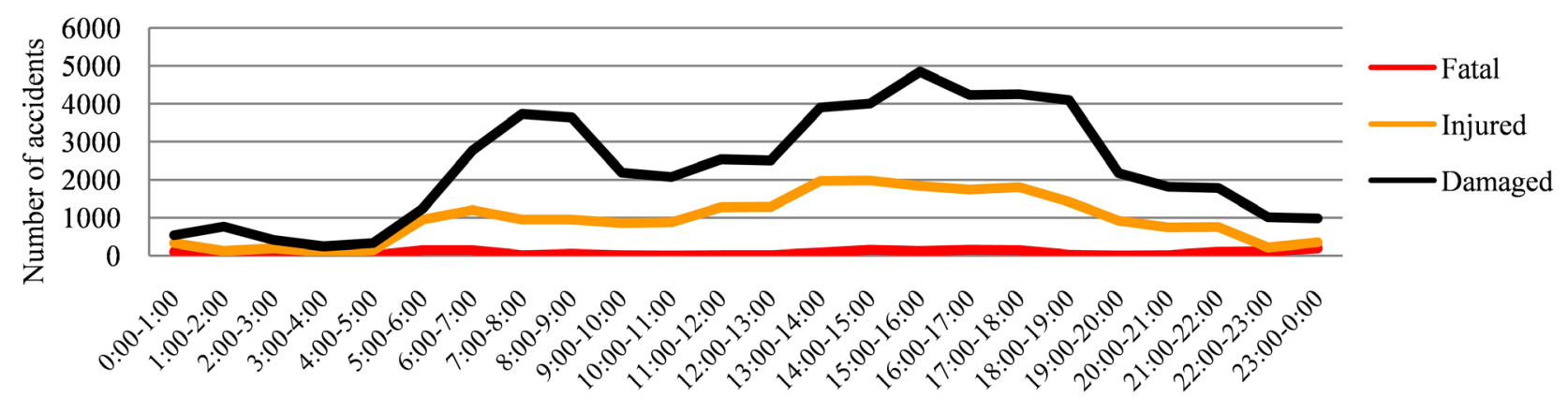

Figure 4. Hourly variation in UTAs type (2011). Source: The Police Department of the I.R.I, Tehran.

\subsection{Hourly Variation in UTA Types}

Comparing the relationship between different hours of the day and the UTA in Tehran, the incidence and intensity of UTAs correlate positively with peak hours. The UTA rate is higher during the morning when offices, schools, and commercial centers open and then during the peak hour in the afternoon when offices, schools, and other government centers close. This is because of the intensity of trip production for many different purposes, especially work and study.

There are two RHs in the morning hours between 7:00 and 8:00 and 8:00 to 9:00 and one $\mathrm{RH}$ from noon to afternoon at 14:00 to 16:00; finally, there is one $\mathrm{RH}$ in the evening between 16:00 and 19:00. During the $\mathrm{RH}$, the traffic is high as a result of urban trips and human movement. Public employees, governmental offices employer, some industrial workers and many students leave their home at the same time. The late afternoon RH and UTAs congestion is again caused by the return trips of the same persons. In addition, most shopping and entertainment trips occur in the afternoon and in the evening. However, the RH for FAs is different from the losses and injury caused by the kinds of UTAs. During this study period, 819 people lost their lives because of UTAs in Tehran City. Of these, 491 were killed in the evening hours and early morning from 22:00 to 6:00. In addition, the largest number of mortalities was among car passengers. 


\subsection{Black Spots over Time}

UTA black spots are usually defined as specific locations in a transportation network with a (relatively) high UTA rate. In order to distinguish these hazardous locations, first, one needs to know where these locations are and then determine the probability of a UTA according to environmental characteristics (e.g., the dominant road type, land use category, etc.). One of the most important purposes of UTA black spots analysis in this study is to discover and generate the information and reasons for these hazardous locations. The information created can be a key to identifying black spot areas and making decisions using empirical scientific and scientific measures to reduce UTA occurrences. Generally, in this study UTA statistics from the Tehran Police are considered as assessment indicators that predict future UTAs and increase safety. By analyzing actual reliable GIS dataset, future patterns of UTAs become more reliable and predictable. In this study UTA hotspots were calculated and analyzed by the KDE in UTA data along Tehran's entire transportation network. Figure 5 shows the results of kernel density application in the study area.

According to the findings, UTAs and hotspots/black spots are dynamic phenomena which are dependent of urban dynamics, urban structure, and time as a crucial factor. Hotspot locations change over time. As Figure 5 shows, high-risk areas in the morning are located in the suburban areas as many highways are located there, and suburban highways are used for connecting suburban to urban zones. As people use suburban highways in the early morning and late at night for starting daily activities and the afternoon for finishing work and returning home, hotspots in the morning period of 0:00-6:00, and the night period of 18:00-0:00, are located in the suburban areas. However, when considering the daily activity of the city, the number and location of hotspots changes.

\subsection{High-Risk Areas}

UTAs occurring close together spatially may be the products of locations with higher levels of conflicting volume, such as commercial land use or some specific road type, such as highways. Identifying high probability UTA areas is an important issue for traffic safety programs. Despite a stricter "black spot" definition in highway safety, UTA-prone locations are regarded as "hotspots," which can be defined differently according to the focus of the analysis.

A considerable number of studies are available on the detection of UTA hotspots, which have been widely discussed in the literature. There are many UTA hotspot analysis techniques used such as KDE, Network KDE, NNH Clustering Algorithm, Black Spot Analysis, etc. There are distinctions made between these methods; one method uses the actual location of a UTA (point in GIS) and one uses the number or density of UTAs for a small geographical area, such as zone or grid cell. KDEs all use zone data while on the other hand, NNH and Black Spot methods use point data. The methodology of spatial analysis can change according to the aim of the research and expected results. For this study, clustering 

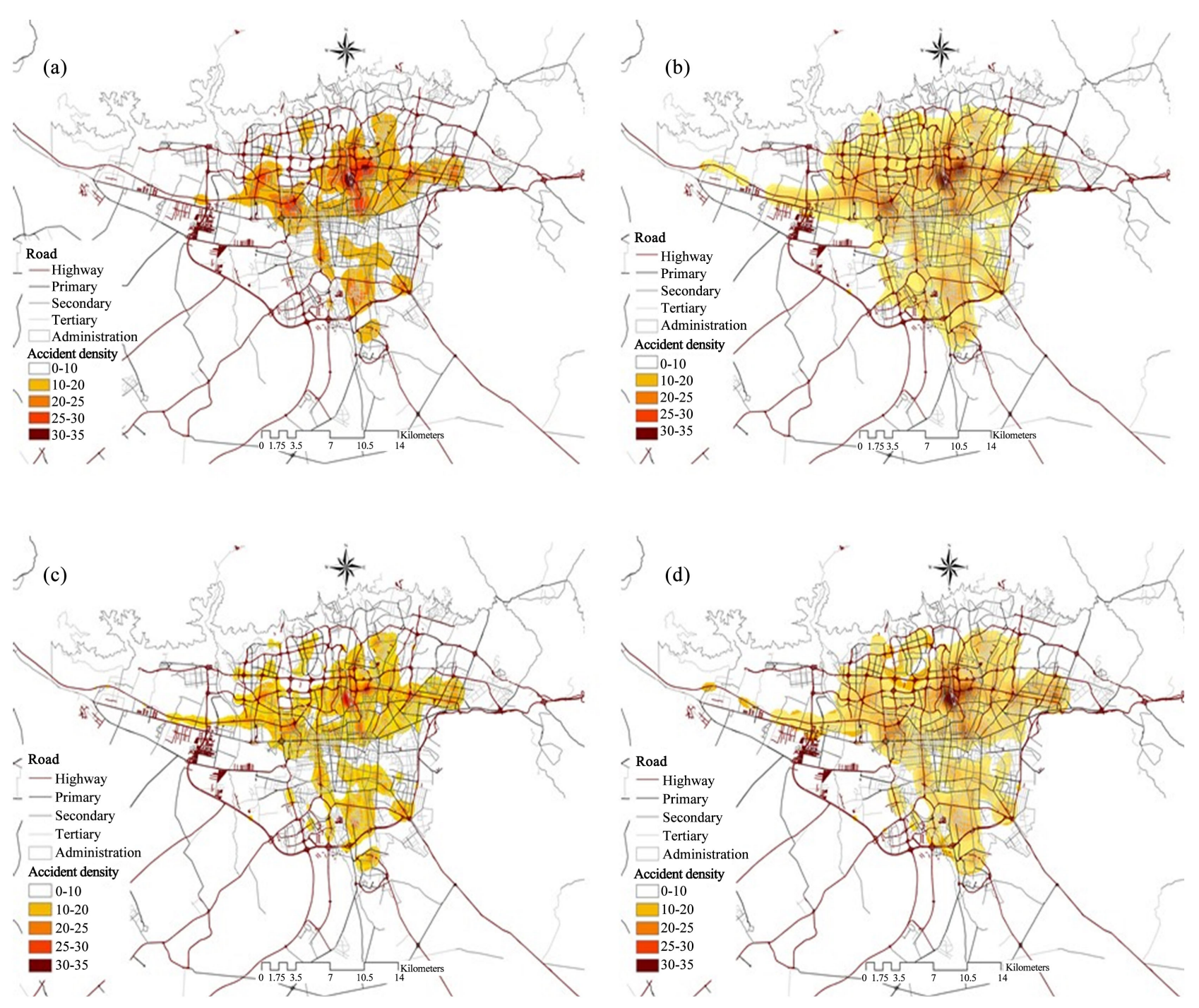

Figure 5. Hot spots of accidents in Tehran city (a) 07:00-09:00; (b) 12:00-14:00; (c) 14:00-16:00 (d) 18:00-20:00.

is used; however, one of the most widely used methods in traffic safety, the KDE method is also performed.

\subsection{Accidents by Land Use}

Land use category and distribution of activities are two main factors that are influencing UTA patterns. Demand for travel is derived from the distribution of work facilities, schools and universities, entertainment and shopping centers, etc. Land use category is a principal factor for urban daily trip contributing and main influencing factors for environments in the transportation network [10]. Ben-Akiva and Bowman [11] stated that land use is one of the major factors in the generation of traffic and UTAs as well. They also indicated that land use is the main influencing on safety and traffic congestion and volume. Today, Tehran residents travel between different districts for different purposes including work, study, shopping, etc. This urban movement and its dynamics cause inte- 
raction between land use systems and transportation networks in different ways. In general, different environments with different geographical features and land use patterns generate different number of urban daily trips. As the number of urban daily trips increase the probability of UTA occurrences with lower severity also increases. The dynamics of the city and accessibility within the city affected by US and land use patterns. In Tehran City, approximately 20,000 - 30,000 cars enter Tehran from suburban cities and, in total, more than 18,000,000 urban trips are made in a day [12]. Number of hot spot by land use category shows in Table 3.

Table 3. Number of hot spot by land use category.

\begin{tabular}{cccccccc}
\hline $\begin{array}{c}\text { Land use } \\
\text { Category }\end{array}$ & $\begin{array}{c}\text { Total number } \\
\text { of } \\
\text { accident }\end{array}$ & CBD & Urban zone & $\begin{array}{c}\text { Suburban } \\
\text { zone }\end{array}$ & Rank & $\begin{array}{c}\text { Hot } \\
\text { cluster }\end{array}$ & $\begin{array}{c}\text { Area of } \\
\text { hot } \\
\text { clusters }\end{array}$ \\
\hline Administration & $3285(5.1 \%)$ & $1.8 \%$ & $3.3 \%$ & $0 \%$ & 4 & 120 & 29.8 \\
Agriculture & $128(0.2 \%)$ & $0 \%$ & $0 \%$ & $0.2 \%$ & 10 & 2 & 4.2 \\
Commercial & $4702(7.3 \%)$ & $2.1 \%$ & $4.2 \%$ & $0.9 \%$ & 3 & 121 & 31.4 \\
Education & $1030(1.6 \%)$ & $0.4 \%$ & $1.1 \%$ & $0.1 \%$ & 8 & 11 & 18.5 \\
Health & $579(0.9 \%)$ & $0.2 \%$ & $0.65 \%$ & $0.05 \%$ & 9 & 9 & 11.2 \\
Industry & $5669(8.8 \%)$ & $0.04 \%$ & $2.6 \%$ & $6.16 \%$ & 2 & 147 & 14.8 \\
Transportation & $1159(1.8 \%)$ & $0.4 \%$ & $0.9 \%$ & $0.5 \%$ & 6 & 114 & 10.6 \\
Park & $1481(2.3 \%)$ & $0.9 \%$ & $1.1 \%$ & $0.3 \%$ & 7 & 51 & 16.4 \\
Residence & $43,613(67.7 \%)$ & $2.6 \%$ & $44.63 \%$ & $20.47 \%$ & 1 & 354 & 34.2 \\
Non-Built up & $2770(4.3 \%)$ & $0 \%$ & $0.2 \%$ & $4.1 \%$ & 5 & 115 & 17.1 \\
Total & $64,421(100 \%)$ & $8.43 \%$ & $58.68 \%$ & $32.88 \%$ & - & 996 & 188.2 \\
\hline
\end{tabular}

Tehran City is face to special pattern of urban dynamics with around 18 million urban trips in a day [12]. These trips consist of traveling for work, education, shopping, and recreation, and are categorized according to trip purpose.

Understanding the relation between accident hotspots and urban structure or built-up environments can be studied on two dimensions. First, it is necessary to identify the distribution and pattern of hotspots across urban structures to determine priority areas where precautions initially take place. As different urban land use creates different population density, speed profile, traffic volume, and pedestrian activity, it is important to divide urban regions into different zones.

In the second dimension, UTA hotspots in Tehran should be analyzed and studied over time to take into consideration the urban network components. As time is the crucial factor in urban dynamics in terms of UTA occurrence, the patterns of hotspots over time are significant. Hotspot analysis is crucial to define where more UTAs take place in order to warn drivers and pedestrians. Different land use areas, such as the CBD, create a non-uniform geographic distribution of UTA locations (and hotspots) over the space. A simple way to asso- 
ciate UTA hotspots (also called "clusters") with urban land use is to divide the urban region into zones with different transportation and land use patterns (Table 4). Traditionally, mature urban regions include, but are not limited to zones classified as CBD, urban zones (or inner city), and suburban zones (or outside zone).

Studying the hotspots or risky areas for traffic safety in Tehran is important to identifying the type of land use in urban areas (Figure 6). As the CBD is an attraction center in the city in terms of business activities, it is important to define it. In addition to the CBD, it is important to define the inner city or urban zone, which can be considered the area highly populated by settlements and urban facilities. By and large, the main entrance corridors are not located in the inner city. For this reason, suburban zone areas should be separated from other zones as the entrances to the central city are located there. Furthermore, the urban transition zones where the high-speed corridors are located should be identified. The location of facilities and their dependence on land use is a reflection of socio-economic and ecological forces. In Tehran City, the growth of urban communities is facilitated by the increase in population and accompanied by the growth of different land uses. These have led to the arrangement of people's activities, buildings, and commercial points in different locations along the transportation network, which in turn generates different trips on a daily basis. The conflict between the potential for population increase and growth of land use results in an increase of daily urban trips that produce UTAs in the urban area. The relationship between land use and UTAs in Tehran is further complicated by increase in mixed land use, and the associated intensification and diversity of lifestyle. The trips generated tend to be high in one place and moderate to low in some places. Moreover, the continuous movement of people on two and four wheels creates UTAs. Figure 7 shows the spatial pattern of UTAs over time by land use. In the morning period, from 0:00 to 06:00, most UTAs occurred in suburban areas. This is because suburban residents use suburban roads for going to the urban areas for daily activities.

Table 4. Ranking hot risky zones cluster by land use.

\begin{tabular}{|c|c|c|c|c|c|c|c|c|c|c|}
\hline County & Rank & $\begin{array}{c}\text { Hot } \\
\text { cluster }\end{array}$ & $\begin{array}{l}\text { Area of hot } \\
\text { clusters } \\
(\mathrm{km})\end{array}$ & $\begin{array}{l}\text { County } \\
\text { area } \\
(\mathrm{Km})\end{array}$ & $\begin{array}{c}\text { Area } \\
\%\end{array}$ & $\begin{array}{c}\text { Area of hot } \\
\text { cluster } \\
\%\end{array}$ & $\begin{array}{c}\text { Hot cluster } \\
\text { dominant land } \\
\text { use type }\end{array}$ & Zone pop & $\begin{array}{c}\text { Pop } \\
\%\end{array}$ & Black time \\
\hline CBD & 3 & 21 & 5.72 & 59.8 & 8.2 & 9.56 & Commercial & $2,408,211$ & 19.4 & $\begin{array}{c}8: 00-10: 00 \\
14: 00-16: 00\end{array}$ \\
\hline Urban zone & 1 & 214 & 58.31 & 391.2 & 53.6 & 14.90 & Residential & $5,983,288$ & 48.2 & $\begin{array}{c}7: 00-9: 00 \\
16: 00-18: 00\end{array}$ \\
\hline Suburban zone & 2 & 132 & 35.96 & 278.8 & 38.2 & 12.89 & Industrial & $4,021,961$ & 32.4 & $\begin{array}{c}\text { 6:00-8:00 } \\
18: 00-20: 00\end{array}$ \\
\hline Total & - & 367 & 100 & 729.1 & 100 & 37.35 & - & $12,413,460$ & 100 & - \\
\hline
\end{tabular}




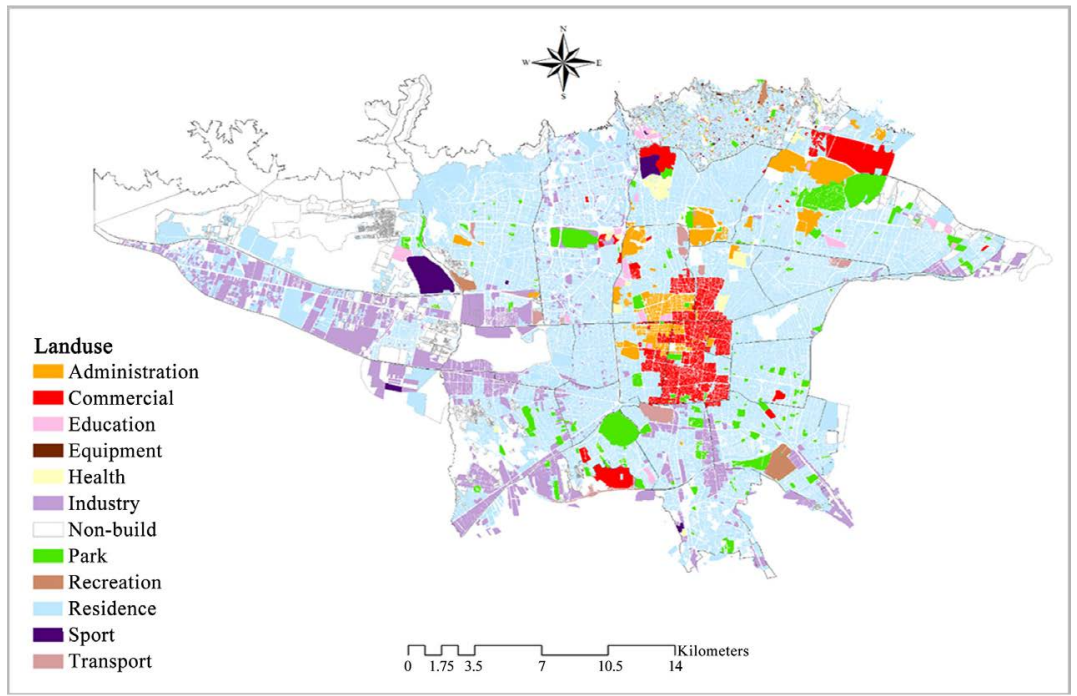

Figure 6. Tehran geocoded land use (2011). Source: Tehran map, TehranCity Hall.
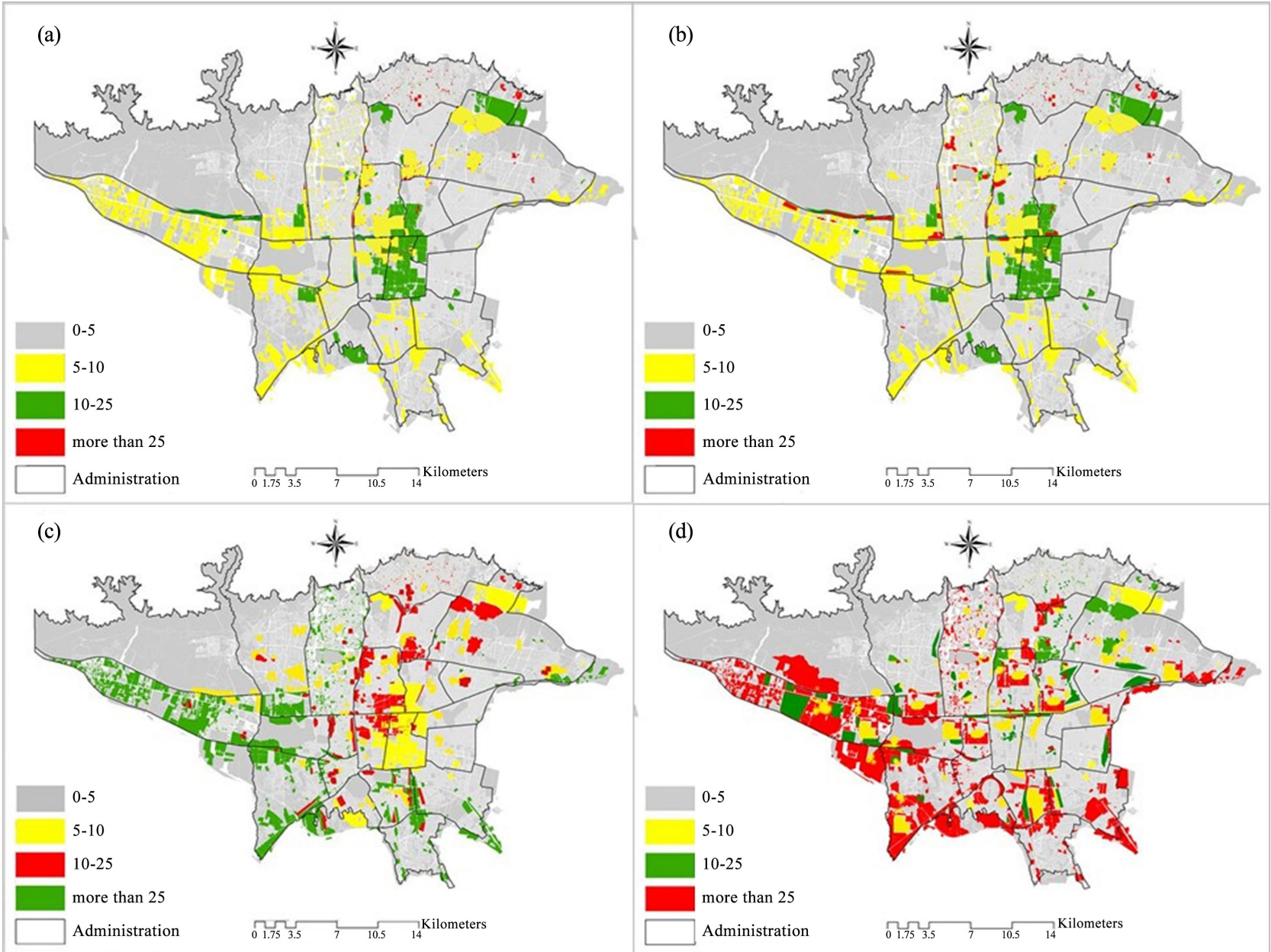

Figure 7. Hot area of accidents in Tehran City by land use (a) 7:00-9:00; (b) 12:00-14:00; (c) 14:00-16:00; (d) 18:00-20:00.

Over time, UTA patterns also change across the city, from the suburban to the urban zone as urban zones are administrative, educational, and commercial 
ports. As a result, UTAs concentrate in the urban zone in the morning period between 06:00 and 12:00 and from the afternoon to evening or 12:00-18:00. From 18:00 people tend to return home by using the highways and freeways in suburban areas, and the UTA pattern moves from urban to suburban zones.

In this study, cluster analysis is used as one of the main methods not only as a spatial analysis tool but also as a statistical method for classifying similar observations. I used this method in order to classify all UTAs in a polygon in to groups as clustering analysis method is one of the most trusted technique for hot spots identification.

The results shown in Figure 7 and clearly indicate that the number of UTAs changes by land use categories over time (see Table 5). In the period of 0:00 to 6:00 and 18:00 to 24:00, UTAs are denser in industrial i.e., suburban areas. The classification is based on the number of UTAs, 0 - 5 UTAs classified as the safest area and more than 25 classifies as the most dangerous. The analysis of UTAs by cluster analysis and over time shows that pattern and number of UTAs change over time by land use. From 6:00 onwards, UTAs move from suburban to urban zones as the urban zones are centers of commercial, administrative, and educational land use and people use them to undertake daily activities. Many people live in the suburban zone and work, study, etc. on CBD and urban zone, there for they move from suburban zone to urban zone every day. As 8:00 is the start of all public facilities and administration offices, people who love in the suburban zone need longer time to reach to the urban zone by time, therefore it was observed that suburban zone has high number of accidents in early morning and late night. The CBDUTAs are shown to occur in the periods between 6:00 and 12:00 and 12:00 to 18:00 because of increasing population and traffic congestion in these time periods. Table 5 shows the most dangerous land use category in Tehran City by the number of UTAs over time. The CBD and inner zone are the riskiest zones while the suburban zone has the highest risk in the period of 0:00 to $06: 00$ and $18: 00$ to $24: 00$.

Table 5. UTAs by land use over time.

\begin{tabular}{|c|c|c|c|c|c|c|c|c|c|c|c|}
\hline \multirow{2}{*}{$\begin{array}{l}\text { Land use } \\
\text { Category }\end{array}$} & \multirow{2}{*}{$\begin{array}{c}\text { Total number } \\
\text { of accident }\end{array}$} & \multirow{2}{*}{ 0:00-6:00 } & \multirow{2}{*}{$6: 00-12: 00$} & \multirow{2}{*}{$12: 00-18: 00$} & \multirow{2}{*}{ 18:00-24:00 } & \multirow{2}{*}{ 7:00-9:00 } & \multirow{2}{*}{ 14:00-16:00 } & \multirow{2}{*}{ 17:00-19:00 } & \multicolumn{3}{|c|}{ Accident type } \\
\hline & & & & & & & & & Fatal & Damaged & Injured \\
\hline Administration & $2639(4.1 \%)$ & 131 & 1215 & 1179 & 402 & 714 & 1041 & 310 & 112 & 1974 & 607 \\
\hline Agriculture & $137(0.2 \%)$ & 79 & 19 & 34 & 5 & 2 & - & - & 2 & 64 & 13 \\
\hline Commercial & $4069(6.3 \%)$ & 249 & 941 & 1956 & 923 & 860 & 1201 & 1425 & 98 & 3781 & 190 \\
\hline Education & $418(0.6 \%)$ & 10 & 221 & 110 & 77 & 154 & 194 & 51 & 21 & 351 & 54 \\
\hline Health & $971(0.9 \%)$ & 92 & 415 & 351 & 113 & 321 & 164 & 21 & 114 & 601 & 253 \\
\hline Industry & $5054(7.8 \%)$ & 2104 & 1414 & 605 & 1021 & 1012 & 1242 & 941 & 131 & 4002 & 921 \\
\hline Transportation & $2180(1.8 \%)$ & 951 & 314 & 101 & 814 & 320 & 216 & 510 & 32 & 1921 & 11 \\
\hline Park & $2143(3.3 \%)$ & 314 & 641 & 619 & 569 & 322 & 219 & 1002 & 57 & 1751 & 227 \\
\hline Residence & $44,796(69.5 \%)$ & 6051 & 12,145 & 13,472 & 13,101 & 3140 & 4151 & 4569 & 148 & 34,521 & 10127 \\
\hline Non-Built up & $2014(3.3 \%)$ & 818 & 215 & 412 & 569 & 816 & 945 & 347 & 54 & 1574 & 386 \\
\hline Total & $64,421(100 \%)$ & 10,799 & 17,540 & 18,488 & 17,594 & 7661 & 9373 & 9179 & 819 & 50,813 & 12,789 \\
\hline
\end{tabular}


Variations in UTAs were examined for each hour in the 24 hours of the day, for four different time periods and 4 rush hours extracted based on the UTAs (07:00-09:00, 12:00-14:00, 14:00-16:00, 18:00-20:00). The results were different and separate UTA distributions.

\subsection{Accidents by Population Density}

An essential indicator of socio-economic identification is population density. An examination of the population density in Tehran City shows the obvious differences between the northern and southern districts of Tehran City.

Tehran population density has the special pattern as population density increased in recent years and the price of land and housing completely different within the districts. The southern districts land and housing are cheap, therefore most of immigrants live there and these areas are the most populous while north area has the lower density as these areas are the most the most expensive areas. According to the census 2011 calculations, District 2 and District 5 with 434 and 402 people/ hectare, respectively, are the most populous districts in Tehran City while district 22 has the lowest which has 1.3 people/hectare. Table 6 and Figure 8 show the result of accidents by population. Accidents by 1000 population result shown in Figure 8.

As mentioned in the literature review, there are many hotspot techniques that can be used. In this study, the NNH clustering analysis method is used for detecting hotspots as it is focused on the identification of groups of data that are spatially close. This particular method's qualification for use is the Euclidean distance between every data point. If a threshold distance is selected, the point pairs with smaller distances are clustered together. The NNH clustering algorithm does not generate clusters from all observations in the study area. As it depends on criteria with regard to distance, only points that satisfy the criteria are clustered.

The simplest way of measuring drivers' behavior, knowledge, and culture is to ask them how they typically act, and thus use individuals as their own monitoring devices. In this way, any behavior, thought, attitude, or experience can be recorded for any time period. One of my considerations is identify the relationship between personal characteristics of drivers (attitudes, behaviors, etc.) involved in UTAs, therefore a questionnaire survey was conducted in Tehran City in June 2016. 1500 questionnaires were distributed in the study area and 1135 of them were returned. The questionnaire results revealed that drivers with less education have the potential of accidents occurrence with perform risky driving, and more educated drivers were least likely to be involved in UTAs. Based on the questionnaire result, male have more dangerous behaviors and drive speedier comparing women, not giving priority to others, and so on. Of respondents, $30 \%$ believed that Tehran UTAs were caused by habit or, on the other hand, by a cultural problem. Moreover, they expressed the belief that penalties are the primary prevention factor in causing them to observe and follow traffic regulations. 
In addition, in Tehran City, the major contributing factors to accidents in the majority of UTAs are drivers' behaviors. Negligence is a main feature in UTAs in Tehran. Refusing to give priority to pedestrians and other vehicles, lack of adequate distance between vehicles, lack of attention to going forward, and high speed are the most prominent problems.

Table 6. Ranking hot risky zones cluster by population.

\begin{tabular}{|c|c|c|c|c|c|c|c|c|c|}
\hline County & Rank & Hot cluster & $\begin{array}{c}\text { Area of hot } \\
\text { clusters }\end{array}$ & $\begin{array}{c}\text { County } \\
\text { area }\end{array}$ & $\begin{array}{c}\text { Area } \\
\%\end{array}$ & $\begin{array}{c}\text { Hot cluster } \\
\text { pop }\end{array}$ & Zone pop & $\begin{array}{c}\text { Pop } \\
\%\end{array}$ & Black time \\
\hline $\mathrm{CBD}$ & 3 & 39 & 17.0 & 59.8 & 8.2 & 343,079 & $2,408,211$ & 19.4 & $\begin{array}{c}8: 00-10: 00 \\
16: 00-18: 00\end{array}$ \\
\hline Urban zone & 1 & 224 & 81.7 & 391.2 & 53.6 & 533,987 & $5,983,288$ & 48.2 & $\begin{array}{c}7: 00-9: 00 \\
15: 00-17: 00\end{array}$ \\
\hline Suburban zone & 2 & 105 & 50.4 & 278.8 & 38.2 & 50,500 & $4,021,961$ & 32.4 & $\begin{array}{c}5: 00-7: 00 \\
17: 00-19: 00\end{array}$ \\
\hline Total & & 368 & 149.1 & 729.1 & 100 & 927,566 & $12,413,460$ & 100 & \\
\hline
\end{tabular}

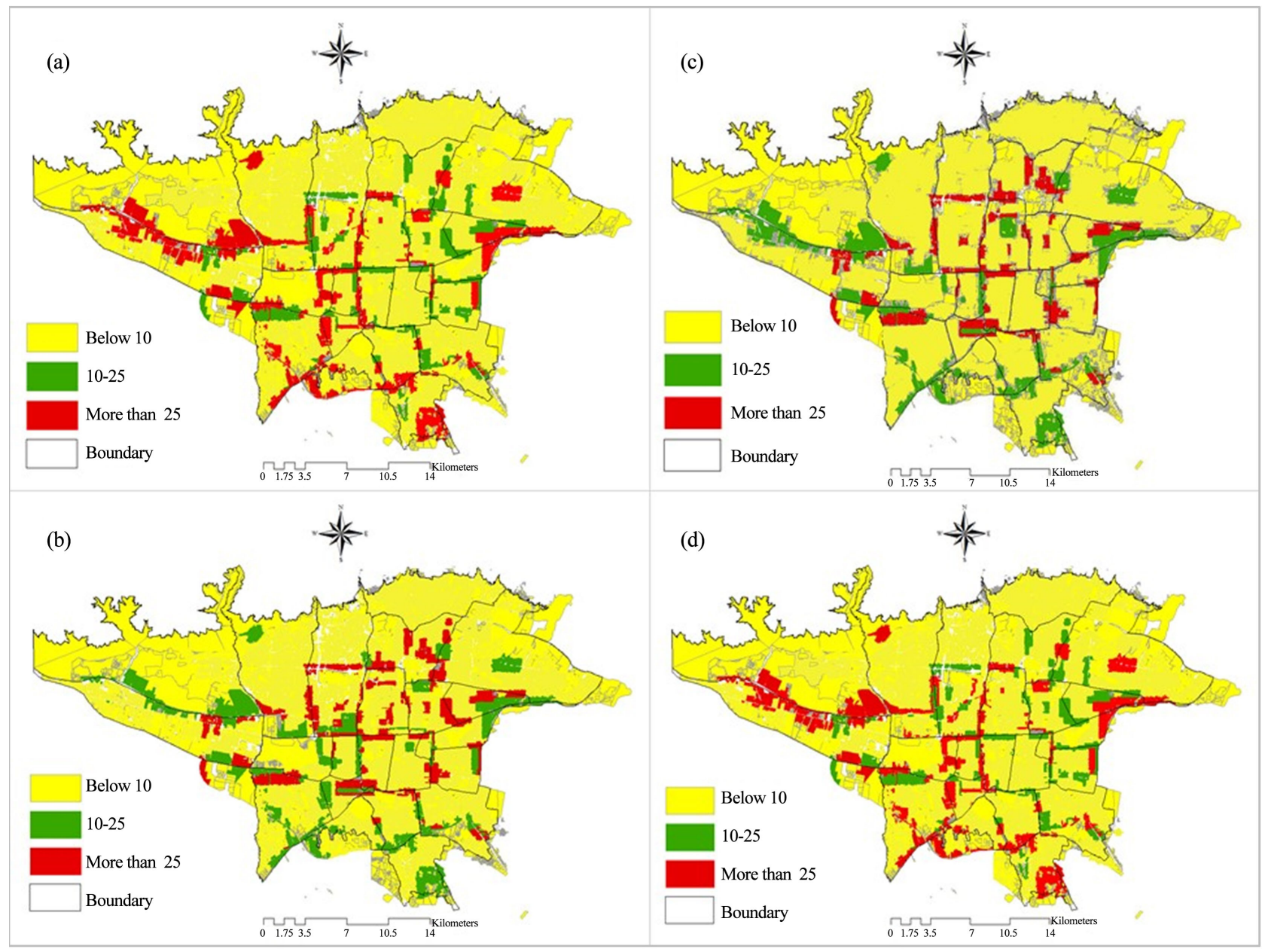

Figure 8. Accidents by 1000 population (a) 07:00-09:00; (b) 12:00-14:00; (c) 14:00-16:00; (d) 18:00-20:00. 
One of the most significant cultural problems in Tehran's UTAs is the movement of pedestrians on roads, without any attention being paid to signage; this also contributes to the incidence of UTAs, which are magnified at road crossings. In Tehran, many UTAs occurred at roads with no space for pedestrians and when traveling on both sides of the road without using the sidewalks. Some other significant cultural problems are: both drivers and pedestrians not paying enough attention to signs, rule violation, a high sense of revenge, legal factors, dealing with offenders, lack of comprehensive rule enforcement that deters offenses, and lack of penalties' inhibiting poor behavior.

\section{Spatio-Temporal Analysis of Tehran UTAs}

The main objective of this study was empirical study of UTAs in Tehran city by urban structure, which is zones, temporally and spatially. For better understanding of spatial pattern of UTAs through the spatial structure of city, the study area divided to 3 zones based on different factors which include but not limited to: 1- Tehran land use 2- Tehran's master plane 3- Tehran's socio economic activities pattern. The 3 zones comprised spatially and temporally for better understanding the effect of urban structure on UTAs. Spatio temporal analysis of Tehran's UTAs shows significant differences through the US. UTAs pattern vary by urban zone, temporally and spatially. Figure 9 shows the variation of UTAs by month; day and time in different zones and express different zone have different patterns and numbers of UTAs. Comparing the UTAs within the zones showed that the intensity and incidence of UTAs are positively correlated with the time and space. Something which accounts for these differences is the US and urban dynamic.

UTAs pattern vary by month and day as well. For CBD zone, March and September, Sundays and Mondays are the black months and days. Comparatively, March and September for urban zone, July and August for suburban zone are the months with the highest number of UTAs in the city, Saturdays, Sunday and Thursdays for urban zone, Thursdays and Fridays for suburban zone are the black days. This could mainly due to the effect of time and culture in urban dynamic. The occurrence of UTAs can vary within the 24 hours of a day across zones due to the urban dynamic. The number and severity of UTAs vary across zone. 09:00 and 18:00 are the black hours for CBD zone while 07:00 and 16:00 for urban zone, 6:00 and 15:00 for suburban zone are the black hours. From a spatial perspective, there are noticeable geographic variations in Tehran's UTAs by zone.

The distribution of UTAs in Tehran's CBD clearly reveals that their nature and origin are entirely different from other zone.CBD with the share of $54 \mathrm{~km}$ in highway, $159 \mathrm{~km}$ in primary roads, $254 \mathrm{~km}$ in secondary road and $212 \mathrm{~km}$ tertiary road with 4792 accidents, is the safest zone in Tehran city. As the CBD is an employment center that attracts far more trips per square kilometer than any of the other zones, the mobility of pedestrian activities is higher when compared to 


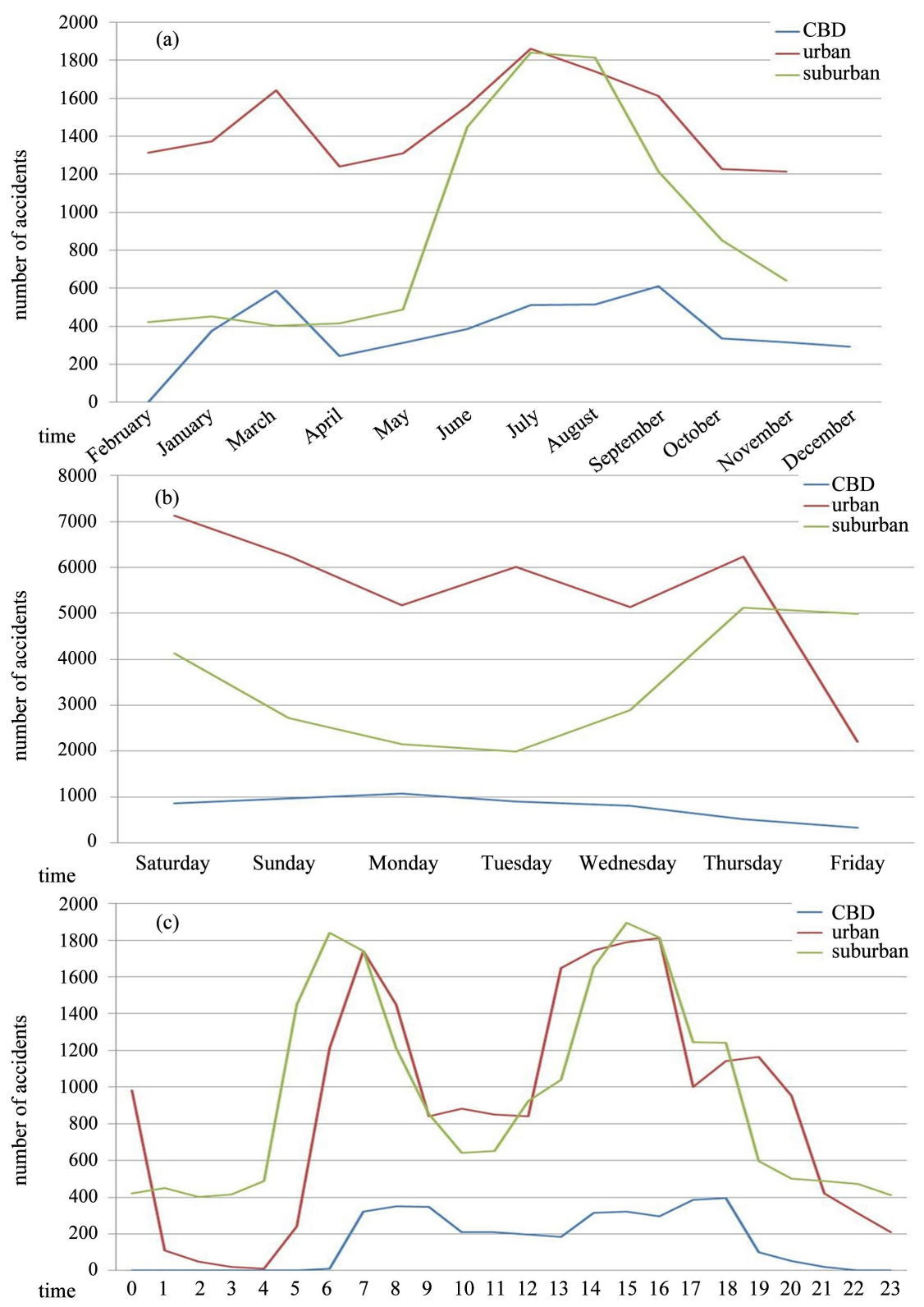

Figure 9. Accidents by (a) month, (b) day and (c) hour in Tehran's zone.

the other zones, but traffic limitations, a contemporary subway system, and an extensive penetration area make this zone safe. "Any movement in Tehran should be planned based on traffic zones. There are two different types of traffic zones in the city:

1) Restricted zones, based on cars' registration number (for odd and even days).

2) Central restricted zones, which is open for public cars (Buses, Taxis, Ambulance, etc.)" (Tehran City Hall).

The main reason of UTAs in CBD zone is penetration mistakes while the main accident type is motorcycle to pedestrians, as this area has the large volume of pedestrians and motorcycles in a day. In Tehran CBD, motorcycle is domi- 
nant vehicle as this area has traffic limitation boundary and cars cannot enter to the zone without official permission but motorcycle can move easily and come to the traffic limitation boundary even without permission. Also based on the structure of this zone, motorcycle is easy to drive instead of cars. In this zone, motorcycles work as a public transportation and many people used these transportation type such as a taxi or bus. The black days of CBD zone are Sunday and Monday, the reason is not completely clear but based on the activity's culture usually people postpone their leisure to CBD from Saturday (first day) to Sunday and Monday as they think the first day is very busy. Also as Friday is weekend holiday and people did some entertainment and leisure on this day, then they prefer to stay at home a day after weekend.

Tehran's urban zone or the inner side of the city faces a different pattern of UTAs between the city (urban zone and CBD) and the suburban zone. Tehran's transportation network has a radial pattern of roads that radiate outwards. Roads are classified on the basis of their location, importance (land use they serve), width, and length, traffic volume, and loads transported [13]. The main type of roads in Tehran's transportation network is categorized as highways, primary roads, secondary roads, and tertiary roads. These roads types have a direct effect on traffic flow, congestion, and UTA pattern.

Many people live in Karaj city (one of the biggest country side city) and other surrounding cities but work or study in Tehran City and thus take roundtrips from Karaj to Tehran. This difference between place of residence and daily life involving work and study creates a special pattern of urban dynamics, urban daily trips, and UTAs. These differences clearly show the effect of socio-economic activities on the equality and quantity of UTAs, traffic volumes, and traffic patterns. Moreover, Tehran's religious activities as well as social activities, and some sporting events take place in the biggest and most famous stadium of Iran (Azadi Stadium), and some special events, such as Ramadan, reveal distinct patterns of when and where UTAs occur within the city. Driving knowledge, culture, and behaviors such as work, school, and socializing affect UTAs [14]. The social, economic, and cultural activities influence the spatial and temporal arrangement of the UTAs in Tehran. Spatial analysis of UTAs by using GIS, discovering where and when UTAs occur, might allow a greater understanding to be reached. Understanding the patterns and reasons for UTAs influences decisions concerning improved and safer roads. The results reveal that more FAs occurred in less urbanized and populated areas, with more highways and wide roads. My finding showed those FA hotspots in areas with low traffic volumes and fewer built-up areas, more highway and freeway road types, converging roads with less police control, and higher speed limitation areas; they are also less urbanized and have lower concentrations of activities and thus low volumes of traffic. Spatial distribution of UTAs in Tehran city reveled that UTAs are more highlighted from the West part of the city toward South which is from Azadi Street to Kazaneh. This pattern caused by the location of two main and 
biggest passenger terminal which are located one in the west and the other in the south and along the highway. These two facilities clearly states how US associated with UTAs.

The findings revealed that the highway and freeway road type with high speed levels have higher rates of FAs and accidents involving injuries, a blacker spot. In Tehran city, main and most usable Highways located from East and southern entrances of the city and continued within the city. More populous areas, such as the urban zone or inner city, were consistently identified as hotspots for accidents involving injuries, while the CBD, as the most populous and dynamic area, was identified as the safest zone with the fewest hotspots. This pattern clearly shows that more urbanized areas with a high density of buildings and populations have a significant traffic volume in comparison with the suburban zone, therefore the severity of accidents is different. The number of hotspots and severity of accidents increases as drivers' speed increases. Results revealed that FA hotspots contributed in highways and freeways where people drive faster; and do match which come from their internal emotions and culture. The results revealed that an area with higher population and employment density, and fewer highways, freeways, and traffic limitation zones will be inclined to have fewer FAs and, in contrast, more UTAs can be seen in areas with more highways and freeways. Fewer UTAs in areas with fewer highways and freeways may be caused by two main factors: the first is intense police control, and the second is more careful driving at lower speeds plus police monitoring. The severity of accident decreased according to traffic volume and city activities. More congested roads are equal to more UTAs but less severity. Traffic volume has an effect on type and severity of accidents as it has an effect on speed by reducing congestion. By using temporal and spatial analysis, I found that in Tehran City, more UTAs occurred on Saturday, Sunday, and Thursday based on the zones and geographical features of the environment. For suburban zone, Thursdays and Fridays are the black days based on the number and kind of accidents. In the CBD, Sundays and Mondays were recognized as hot or black days. In CBD, most of the time, especially during the $\mathrm{RH}$,

Regarding time of day, it was identified that UTAs occurred at different times in different zones, as the zones' times for urban daily trip generation are different. Of all UTAs, most occurred between 8:00 and 20:00. The results of spatiotemporal analysis of UTAs in Tehran City show hotspot patterns are changed by time, space, and US. It was found that the incidence of UTAs was because of different factors, the most important of which are time, US (zones), land use, and population, which have interdependent relations with each other. The land use characteristics determine the function of an area and population determines the rate of activities in the area. The land use category has a direct impact on the potential risk of UTAs. In Tehran City, land use category and urban activities, which are typical of different zones, are significantly involved in the kind, type, and rate of UTAs. The UTAs and land use interaction can happen between zones or within a zone. There is a strong possibility and rate of UTAs in Te- 
hran's urban zone because of mixed land use established within the zone. Tehran's urban zone is a combination of residences, health and administration facilities, entertainment, and shopping centers. When land use is defined as having a single purpose, such as industrial land use in a suburban zone, the interaction of land use and transport tends to occur between or within zones.

Some land use categories have noticeably different effects on UTA severity at different $\mathrm{RH}$ and non-RH periods. For example, in the CBD, commercial facilities and the Grand Bazaar act as trip attraction within work time, while most leisure facilities and residential land use activities attract people after working hours. Therefore, different patterns are created in UTAs, according to RHs and non-RHs in the land use categories.

For all fatal results, most of the hotspots are located in the local zone where high-speed corridors and main arterials are mostly located. Land use category and urban density were associated with an increased number of UTAs and serious injuries.

Based on the methodology in this study, time is a crucial factor in UTAs, which has a direct impact on the transportation network congestion level, transportation mode, daily urban activity, daily urban trips, mobility which affect the speed level. During the RH and when the transportation network is congested, decreasing speed reduced the severity of UTAs, which indicated that travel speed is a key factor that is affected by land use category, population, drivers' knowledge, and culture. Period variations clearly show that the black period is changing by land use category and urban zones.

As expected, the distribution of UTAs over an urban region was not uniform. Therefore, it is important to detect UTA-prone locations or hotspots. Even though there is a well-defined "black spot" definition for UTAs, safety practices are not that easy or straightforward to adopt in urban regions. Largely because of the complex road network in urban areas, it is not easy to define a unit segment. For this reason, there is no strict definition UTA hotspots. The definition of hotspots in urban areas can also vary based on the scope of the study.

To address these complications in urban traffic safety analyses, it is important to develop a methodological framework for studying hotspots in urban regions, which is the primary focus of this study. The proposed methodology focused not only on detection of UTA hotspots, but on assessment of their distribution with regard to the built environment's features. ArcGIS, which produces clusters, provides for a hotspot detection process. The built environment relations are analyzed at the level of urban zones and distance to land use. While all the calculations are carried out in a GIS environment, the significance of the relations is tested via statistical tools (Table 7).

The main objective of this analysis was to investigate the spatiotemporal structure of UTAs. To achieve this, the temporal and spatial analyses were conducted for different spatial zones (CBD, urban, and suburban); when these were combined it was found that they were more likely to be distributed temporally. CBDUTAs occurred more frequently in the morning peak. However, more 
UTAs were likely to occur during the afternoon period in urban zones and especially from 14:00 to 17:00. This is due to the high number of workers or shoppers in the city, which causes more frequent pedestrian-vehicle UTAs while the black time for suburban UTAs is after midnight. I also discovered that losses or damage UTAs mostly occurred during the day, while fatal and injurious UTAs are more likely to happen at night. To demonstrate the clustering of UTA frequency, and thereby reveal spatial hotspot areas, KDE was applied. While analysis at a larger spatial scale provides a general overview of the nature of UTAs, it does not reveal underlying trends and patterns that might be beneficial to understanding the characteristics of UTAs by land use and population. Urban zones can be divided into sub-zones to understand the relation between UTA occurrence and land use. Residential, commercial, educational, industrial, and non-urban areas among others can be defined more precisely to understand the relationship between UTA hotspots and land use (Table 8). Furthermore, if available, land use information should be utilized. Analyzing hotspots with land use information may be more informative for traffic safety officials.

Table 7. Statistics of hotspots.

\begin{tabular}{|c|c|c|c|c|c|c|}
\hline \multirow[b]{2}{*}{$\begin{array}{l}\text { Minimum number } \\
\text { of UTA per hot spot, } \\
\text { nmin }\end{array}$} & \multicolumn{2}{|c|}{$50 \mathrm{~m}$} & \multicolumn{2}{|c|}{$100 \mathrm{~m}$} & \multicolumn{2}{|c|}{$200 \mathrm{~m}$} \\
\hline & $\begin{array}{l}\text { Average number of } \\
\text { UTA per hot spot }\end{array}$ & $\begin{array}{l}\text { Average trimmed } \\
\text { hot spot length } \\
\quad \text { (in } \mathrm{km})\end{array}$ & $\begin{array}{l}\text { Average number of } \\
\text { UTA per hot spot }\end{array}$ & $\begin{array}{l}\text { Average trimmed } \\
\text { hot spot length } \\
\quad(\text { in } \mathrm{km})\end{array}$ & $\begin{array}{l}\text { Average number of } \\
\text { UTA per hot spot }\end{array}$ & $\begin{array}{l}\text { Average trimmed } \\
\text { hot spot length } \\
\text { (in } \mathrm{km})\end{array}$ \\
\hline 1 & 4.717 & 0.010 & 5.821 & 0.250 & 6.141 & 0.441 \\
\hline 2 & 5.655 & 0.021 & 6.741 & 0.325 & 7.152 & 0.511 \\
\hline 3 & 5.812 & 0.025 & 6.842 & 0.384 & 7.251 & 0.523 \\
\hline 4 & 6.021 & 0.041 & 7.231 & 0.410 & 7.651 & 0.558 \\
\hline 5 & 7.125 & 0.061 & 8.214 & 0.510 & 8.001 & 0.601 \\
\hline 6 & 7.845 & 0.074 & 8.412 & 0.521 & 8.021 & 0.615 \\
\hline
\end{tabular}

Table 8. Number of hot spot by land use category.

\begin{tabular}{|c|c|c|c|c|c|c|c|}
\hline Land use Category & $\begin{array}{l}\text { Total number of } \\
\text { accident }\end{array}$ & $\mathrm{CBD}$ & Urban zone & Suburban zone & Rank & Hot cluster & Area of hot clusters \\
\hline Administration & $3285(5.1 \%)$ & $1.8 \%$ & $3.3 \%$ & $0 \%$ & 4 & 120 & 29.8 \\
\hline Agriculture & $128(0.2 \%)$ & $0 \%$ & $0 \%$ & $0.2 \%$ & 10 & 2 & 4.2 \\
\hline Commercial & $4702(7.3 \%)$ & $2.1 \%$ & $4.2 \%$ & $0.9 \%$ & 3 & 121 & 31.4 \\
\hline Education & $1030(1.6 \%)$ & $0.4 \%$ & $1.1 \%$ & $0.1 \%$ & 8 & 11 & 18.5 \\
\hline Health & $579(0.9 \%)$ & $0.2 \%$ & $0.65 \%$ & $0.05 \%$ & 9 & 9 & 11.2 \\
\hline Industry & $5669(8.8 \%)$ & $0.04 \%$ & $2.6 \%$ & $6.16 \%$ & 2 & 147 & 14.8 \\
\hline Transportation & $1159(1.8 \%)$ & $0.4 \%$ & $0.9 \%$ & $0.5 \%$ & 6 & 114 & 10.6 \\
\hline Park & $1481(2.3 \%)$ & $0.9 \%$ & $1.1 \%$ & $0.3 \%$ & 7 & 51 & 16.4 \\
\hline Residence & $43,613(67.7 \%)$ & $2.6 \%$ & $44.63 \%$ & $20.47 \%$ & 1 & 354 & 34.2 \\
\hline Non-Built up & $2770(4.3 \%)$ & $0 \%$ & $0.2 \%$ & $4.1 \%$ & 5 & 115 & 17.1 \\
\hline Total & $64,421(100 \%)$ & $8.43 \%$ & $58.68 \%$ & $32.88 \%$ & - & 996 & 188.2 \\
\hline
\end{tabular}


As expected, the number of hotspots obtained from all UTAs shows differences regarding different time and zones. For all results, it can be said that most of the hotspots are located in the third zone where high-speed corridors, primary industries, and factories are mostly located. More than half of the total number of calculated hotspots is seen in the third zone where high-speed corridors and main arterials are located. Traffic safety officials should be aware of this situation as nearly half of the total hotspots calculated are seen in this zone.

Injury hotspots are also calculated by different time and zones. The most striking result to emerge from hotspot analysis of pedestrian UTAs is that most of the hotspots are located in the second zone where settlement areas are mostly seen. As expected, more than half of the total number of hotspots is concentrated in the second zone. This situation may be attributable to the low mobility of pedestrian activities compared to other zones. Fewer UTA hotspots are seen in the CBD. This may be because of strict traffic limitation boundaries in the CBD that only allow some cars to enter this area.

Time-based hotspot locations are also dispersed over the study area. In all periods, the observed hotspots in the $\mathrm{CBD}$ are minimal, with the urban zone and suburban zone having higher than expected values taking into consideration that the uniform distribution of the number of hotspots over the four time periods shows differences. According to this, the observed number of hotspots for morning and night-time periods is lower than expected. In contrast, the found rate is higher than the expected number of hotspots when afternoon off and night-time periods are taken into consideration. The temporal distribution of hotspots shows differences. Accordingly, fewer hotspots are observed when compared to the expected values for peak morning and night-time UTAs. On the other hand, there are more hotspots calculated than expected for afternoon and evening peaks.

In this study, I also tried to examine and explain the relationships between various land use of US and UTA in Tehran city. The results showed that different land uses generate different kinds of urban and human movement, which are undertaken using various modes of transport to reach different activity centers. The connection between traffic flow and land use produce road UTAs in the urban area, which occurs when the interaction between cars, transportation network users, and the roads become defective. Findings showed that land use is not the only determinant of road UTA; others such as increased traffic flow, dangerous driving, lack of concentration, refusal to obey traffic rules, and mechanical faults are also major causes of road UTAs in Tehran City. Evaluation of the results shows that urban zones with commercial and administrative land use, and suburban zone with industrial land use are very dangerous with respect to both fatal and injurious UTAs. This situation may occur because of poor geometric design or inadequate regulations. To provide traffic safety, more effort could be made to develop and manage the zones' structure. When creating or improving the geometry of roads, traffic safety issues should be taken into con- 
sideration. Rather than giving the automobile priority, pedestrian priorities should be considered. On the other hand, especially in Tehran, the speed profile is higher than the intended speeds. This situation is true for all zones in the study area. In the CBD and residential areas, the average speed should be 30 $\mathrm{km} / \mathrm{h}$; however, speed profile is mostly seen at $50 \mathrm{~km} / \mathrm{h}$ and more. This problem arises from the incorrect design of roads. In Tehran, transportation is planned to take into consideration the vehicle's priorities. This can be seen clearly from the nonstop design of the arterial roads. Even in the urban zone, where residential areas are mostly seen, speeds are seen of over $50 \mathrm{~km} / \mathrm{h}$. In the off-peak hours, speeds can reach $70-90 \mathrm{~km} / \mathrm{h}$. High speeds on the arterials roads, especially in the residential areas, results in a great number of UTAs occurring. In summary, it is evident that to provide traffic safety in Tehran, traffic speed and traffic volume should be brought under control by traffic safety officials.

Distribution of UTA hotspots shows that, in the CBD, the number of observed hotspots is less than expected when the total urbanized area in the zone is taken into consideration. As the CBD is an employment center and attracts far more trips per square kilometer than any of the other zones, the mobility of pedestrian activities and traffic volume are higher than other zones, but strict traffic limitation boundaries also make this zone safer. To provide safety, regulations could be implemented in other zones too.

About the analysis of demographic factors based on the Tehran census data and questionnaire results, the age group at highest risk has been extracted. Most drivers who contributed to the fatal and injured categories of accidents range from 19 to 39 years. They also believe that traffic regulation rules cannot prevent them from breaking the rules. Based on the interpreted data, people perceive that current penalties are not strong enough: any one can easily break the rules and pay the penalty, current penalties are inhibitor enough and anybody can easily break the rule and buy it by money. Of course, the penalties do not work at all when they are too inexpensive. In summary it was found that summer sees the highest rate of UTAs but there were not extremely big differences between summer and other seasons.

In the period studied, Ramadan was in August. Changes in sleeping patterns, meals together, fasting during the daytime, and the high rate of urban dynamics in more than one area of life, included sleepiness, increased irritability, a decrease in concentration, and reduction in physical performance. In conclusion, it was found that during Ramadan, changes in meals and rest behavior affected urban dynamics, human movement patterns, and the UTA pattern. Reducing working hours in Tehran as well as in other cities in Iran, and changing work hours have a direct effect on the patterns and number of UTAs in Tehran City. Thus, the rate of road UTAs was lower during the month of Ramadan than in other months.

The minimum rate of UTAs was related to Friday as Friday is the weekend in Iran's calendar. The UTAs occurred mostly between 14:00 and 16:00 in Tehran 
City as most schools, universities, and even administrative offices finish at this time. The study found that land use categories, the physical characteristics of the environment, and urban structure all have an effect on drivers and human behavior. Thursdays' UTA rates are high in Tehran City as they are the last day of the week; $18: 00$ to $21: 00$ is the last period of work on the last working day of the week, and after 21:00 businesses and department stores close.

UTAs are complex events and many different factors have an effect. In this study, it was found that UTA occurrence times and patterns change by urban dynamic, traffic volume, and urban mobility. I have examined 64,421 UTAs that occurred in Tehran in 2011; these UTAs in Tehran City might occur on any day at any time and incur many negative side effects. It was found that the incidence of UTAs over time and over the US, including zones, population, land use, and road types were closely associated with the underlying land use category and geographical characteristics of the area. This study did a spatiotemporal analysis of UTAs in Tehran City and investigated the relationship between UTAs and the US including land use and population in Tehran City. This research also sought to develop a GIS-based approach to performing an area-wide link-based relative accidents risk analysis. The method was used to capture the spatial and temporal patterns of risk factors for UTA and to identify the actual high-risk segments of the traffic network. Cluster analysis and KDE are sufficient to spatially estimate the observed risks with significant variance and high uncertainty, and to investigate the real safety tendencies underlying the UTA data. The KDE method and cluster analysis method were applied in this study as a means of generating relative risk maps and investigating the real areas with high rates of UTAs. Distribution of UTA hotspots shows that, in the CBD, the number of observed hotspots is small. As the $\mathrm{CBD}$ is an employment center and attracts far more trips per square kilometer than any of the other zones, the mobility of pedestrian activities is higher when compared to the other zones; however, traffic limitations, an attractive subway system, and an area of extensive penetration make this zone safe. Regarding the fatal results, most hotspots are located in the suburban zone where most high-speed corridors and main arterial roads are located. The land use category and urban density were associated with an increased number of UTAs and serious injuries.

Time variations clearly show that the black period is changing according to land use category and urban zone. The results of both spatial and temporal analysis show that suburban zones with industrial land use are the main land use type associated with dangerous or fatal UTAs. Moreover, the CBD, with a high rate of urban mobility but a low density of residential areas, is the most dynamic zone and is associated with fewer accidents in number and severity. Temporal results show the variation, patterns, and occurrence of UTAs in Tehran City by season, month, day, and hour. For all of the UTAs per season and per week, summer is the black season based on increasing urban mobility, and fatal UTAs. The latter occur more frequently, and Thursday and Monday are the least safe 
days; these are followed by the weekend (Friday), which is relatively more harmless. This daily pattern clearly shows the effects of urban mobility and urban daily trips on the UTA rate.

Within a 24-hour period, the daytime is less safe than night-time, especially in the afternoon (15:00 to 16:00), which is the least safe time in all days. During weekdays and Fridays, there are two flat risk peaks in the daytime: the morning peak (7:00 to 9:00) and the afternoon peak (14:00 to 16:00). In the UTAs examined in Tehran, the detected hotspots were found to be significant. Regarding traffic safety, the following conclusions were derived from the findings.

It was seen that the UTA density pattern has changed dramatically in various zones and time periods. Hence, any change in the vicinity of road sections should be evaluated in space and time with regard to safety. Shopping malls increase the number of UTAs significantly and therefore increase the UTA risk around them if the surrounding environment is not adapted accordingly. The suburban zone with industrial land use is related to the number of UTAs caused by high speed on the highways.

It was observed that the UTA density pattern changes dramatically in various zones on an hour by hour basis and according to the RHs. More UTAs but with less severity occurred in daytime in Tehran when traffic is congested in the city and crowding exists on the public transportation network. Usually, this is common at two times of the day, first in the morning $\mathrm{RH}$ and then in the afternoon and evening $\mathrm{RH}$, the times when most people commute and the city is particularly dynamic. This condition is very broad and often refers specifically to an urban dynamic when there is a large volume of cars, people are on the road, and there is some disruption to speed.

Different zones have different RHs; this causes the CBD, urban, and suburban zones to have a different congestion pattern and causes UTAs as well. These are due to a large number of people engaging in activities that involve using their cars or public transportation. These motorists are often going to restaurants, administrative, and commercial land use locations in the urban zone, and industrial and cemetery sites in the suburban zone where vehicles are crowded and the area is more dynamic because of its population. The academic areas, which refer to areas with more schools, institutes, universities, and education facilities may have extra volumes of traffic and congestion levels. Thus, the different land use categories and different functions of areas with different RHs in zones contributes to the different spatial pattern of UTAs within the city. On Thursdays, the schools and administrative offices are on a half-day schedule, and many people work only half-time. This leaves more time for amusement, shopping, and any other activities. This causes a different RH on Thursdays as the day just prior to the weekend. It takes some traffic load from the evening $\mathrm{RH}$, and thus makes the morning RH the most intense time of the day. Moreover, the spatial analysis and spatiotemporal analysis of relative UTA risks point to the risky segments of the transportation network for different zones of the city and different land uses, as 
well as seasons, months, days, and times.

This study examined how land use generates crisis-crossing trips using various modes of transport to convey people; it provides an explanation of how the relationship with land traffic flows creates road UTAs in Tehran. It was discovered that the locational pattern of the various land uses in the urban area is a reflection of socio-economic and ecological factors. It was also found that the residential areas are trip or traffic-generating areas, while attraction areas are the activity itself for workplaces. The movement of vehicles carrying people and goods between these regions results in the crisis-crossing of traffic flows, which inadvertently generate road UTAs, along with the other identified UTAs. This study revealed that the rate of the rate of UTAs occurring because of road traffic varies on the designated roads, but is moderate in the urban zone.

The findings of this study can be a useful key to better decisions-making regarding traffic safety and policies to reduce the rate and number of UTAs. The results of this study can be used by the police or other departments that are involved in traffic safety and transportation.

The negligence of drivers is the main feature of the UTAs in Tehran. Refusing priority to pedestrians and other vehicles, lack of sufficient distance or driving too close, lack of attention to prospective dangers and high speed are the predominant problems. One of the most significant cultural problems in Tehran City UTAs is the movement of pedestrians on the roads; by not paying any attention to signage, pedestrians also contribute to the incidence of UTAs, and this is magnified at road crossings. In Tehran, many UTAs occur on roads with no walkways for pedestrians, and when traveling on both sides of the road without using the sidewalks. Some other significant cultural problems are: insufficient attention paid to signage (both drivers and pedestrian), breaking the rules easily, having a high sense of revenge, legal factors, dealing with offenders, rule violation, lack of complete and deterrent rules enforcement, a lack of penalties that inhibit poor practices. Human factors such as the driver's panache, pride, internal stimuli like anger, stress, and excitement, and uninformed drivers can also play a role. Adequate maintenance of cars and vehicles during their working life is another important factor in safe driving. UTAs are frequently caused by old vehicles with mechanical defects as well as new cars with poor maintenance. Yet in Tehran, the majority of cars are new cars; old cars account for the lowest number of vehicles but the quality of new vehicles is a critical factor for which the car production companies are responsible. Unfortunately, neither the government nor the author could obtain any reply from them. The variation of UTAs by season, month and day by zone and the pattern of population movements by accident severity, time of the day and location during the day shown in Figure 10 and Figure 11.

\section{Conclusions}

The main aim of this study was an empirical study to analyze UTAs in Tehran 
City spatially and temporally in order to reveal patterns and significant associated factors of UTAs in the study area and to compare city zones through the US to identify the relationship between the US and UTAs over time. As variation in the distribution of UTAs occurs within a 24-hour period through the US, there is a disparity in UTA frequencies among the different factors, of which land use, population, road types and time were the most associated factors in this study. There is a significant variation in the occurrence of UTAs through the US which is 3 zones (CBD, urban zone and suburban zone), as well as time.

This study has demonstrated the capability of GIS in identifying UTA locations in Tehran City; it was carried out to describe the characteristics of UTAs, their location, their leading causes, their socio-economic impacts in terms of time and space, and to propose appropriate interventions that could help to reduce UTAs. This study can be used to compare different types of UTAs in relation to different times, different urban zones, road variations, land uses, and

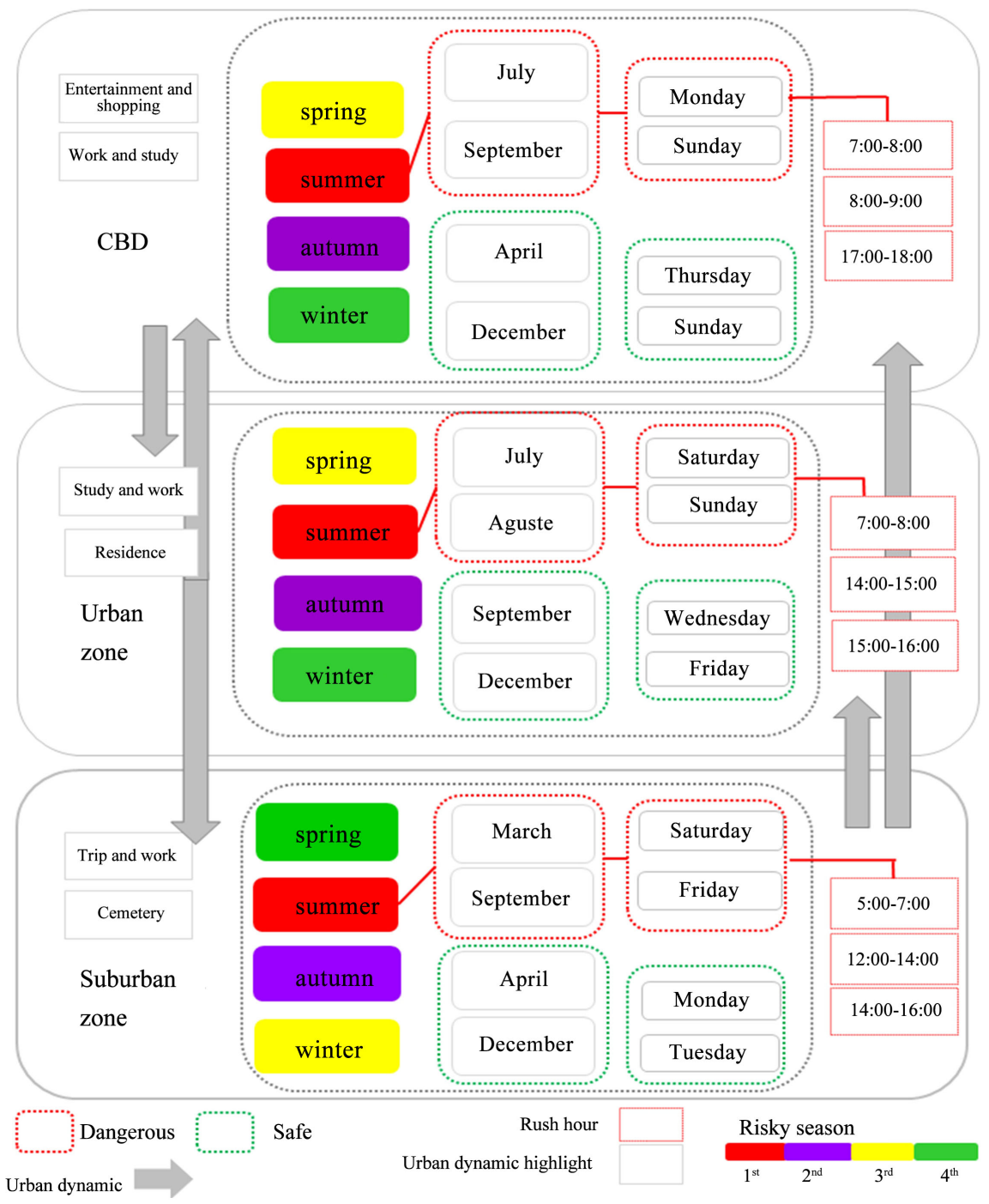

Figure 10. Variation of UTAs by season, month and day by zone. 


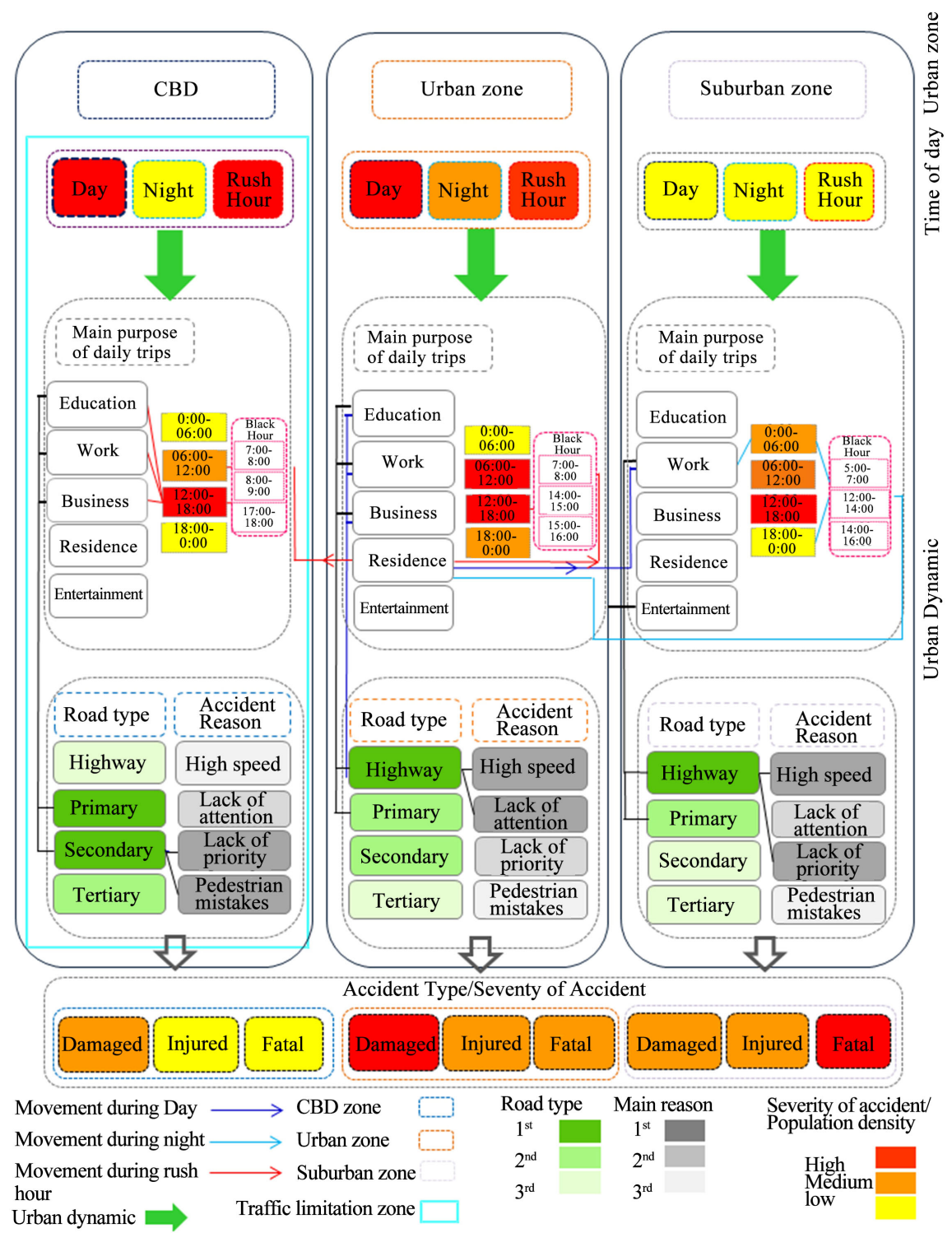

Figure 11. Population movements by accident severity, time of the day and location during the day.

population. Specifically, the work presented the probability of a UTA at different locations in the US. The analysis found that suburban zones and the urban zones with more highways have the highest possibility of fatal and injurious accidents while CBD recognized as the safest zone based on the number and severity of UTAs.

My findings show that UTAs causes are different in different zones; the cause of UTAs can usually be traced to a combination of circumstances in a chain of events that are best described by interpreting the relationships among 1) urban dynamics 2) the transportation network user, 3) the road type, 4) the road environment, and 5) culture. Through the city, based on the US, several areas are considered high-risk areas but CBD is excluded. The majority of fatal and injured UTAs have occurred on highways which are located at the suburban 
zone and main entrance which means the areas in south, east and northwest of Tehran. Hence, highways are the most dangerous road type and suburban zone is the most dangerous zone in Tehran city. The results also show that areas with more highways and freeways that are less urbanized and have a lower population density will tend to have more dangerous UTAs and FAs; in contrast, more urbanized and built-up areas have more UTAs but ones with less severity. This situation is caused by a key factor, namely, speed. In more urbanized areas with greater density of population and buildings, higher traffic volumes and greater police control, such as the $\mathrm{CBD}$, drivers have to reduce speed and drive more carefully regardless of land use category, sometimes, the traffic volume is intense and accidents increase accordingly, but their severity is low. The results indicate that the rate of accidents increased as congestion decreased and speed increased. Safer interaction between US and UTAs is encouraged by lower speed. Based on the methodology of this study, time or hour of day is the most dependent on UTAs type, rate and severity. Types of road variations in different zones clearly show that in contrast to many cities in the world, Tehran's highways and expressways are not the safest for driving. This study found that areas with more urban mobility and population density-the CBD and urban zone-have relatively higher but less severe UTA rates [15].

Various variables like month, day type, time, space, and the structure of the surrounding such as weather and brightness conditions have various impacts on UTA rates and severity; that is why the frequency and occurrence of UTA in Tehran City exhibit different patterns. UTAs are randomly distributed in the city in terms of time and space. The various reasons causing numerous UTAs are lack of priorities among drivers, lack of attention to the prospective danger, high speeds, and pedestrian mistakes.

UTA points with valuable attribute features can act as analytical tools. Using GIS with these data can prove a particular spatial pattern and can also show how the spatial patterns of UTAs may have a spatial dimension (i.e., a relationship between time and the reasons for the UTA results in terms of fatalities, injuries, and losses). This study can be used to compare different types of UTAs according to different time and road variations. More specifically, this work presented the probability of a UTA at various locations in the road network. From the analysis, it was found that highway and freeway, residential, and secondary road types are those with the highest possibility of a UTA. The primary causes and contributing factors of UTAs in Tehran have been treated by using the UTAs data from the Tehran Police and the original data obtained from questionnaires and field work. Tehran, as the capital of Iran and one of the most important cities not only in Iran but also in the Middle East, and the center of many national and international organizations, has experienced a rapid growth in its urban population.

The increase in the expansion of the city boundaries, and the limitations and 
lack of sufficient public transport (even though there is a high incidence of urban transportation and high use of cars for daily urban trips), inadequate sidewalks, and problematic driving culture, plus a large number of UTAs, makes the Tehran UTA problem severe. There is a high concentration of government administrative facilities and institutions, commercial, educational, and business activities mixed with residential areas and residential land use in Tehran, especially in the center. Tehran City is also characterized by the most advanced traffic control systems and regulation in Iran, but the lack of penalties to reduce the high rates of violation and the absence of a sufficient driving culture as well as the lack of current road transport regulation has resulted in a significant number of UTAs. This study considered the primary causes and contributing factors in Tehran UTAs. In this study, road types and some facilities are treated separately. Time is also considered as a crucial factor and this study contributes to a detailed understanding of the spatial and temporal characteristics of UTAs in Tehran City. The land use category was found to have an impact on socio-economic patterns and thus the type and severity of UTAs. The results showed that all Tehran zones have a trip or traffic-generating role, but this role change over time. Every zone and special land use category has an urban trip production role but this role changes across zone according to time. My findings suggest that more urbanized area with higher population density with fewer numbers of highways, freeways, and traffic limitation zones will tend to have fewer dangerous accidents i.e., FAs, particularly less urbanized area with more highways and freeways have more UTAs. It caused by first lower speed which cause by congestion and high traffic volume. The next issue caused by more urban and socio-economic activities. Considering the accident data in this study, travel time may be more dependent on congestion, road qualities, and geometric elements than posting speed limits on signs. Road type variations clearly show that in contrast to many cities in the world, Tehran's highways and expressways are not the safest for driving. Area with high socio-economic and urban activities are tend to have more UTAs rate but with lower severity [15] road length also have effect on UTAs rate and severity. UTAs in wider roads have more serious injuries, especially for highways. Time series data would also allow further analysis of changes over time and can avoided the problems.

In addition, according to the number of accidents in Tehran and percentages of driving violations, it is really important to make more preventive laws and rules. It seems that current laws are not effective enough. To ensure more effective control, the police should contribute to providing new laws and regulations. In addition, the penalties are not sufficiently high and most people can pay fines easily. This means that offenders can easily "buy" a violation of the law. On the other hand, the existing traffic regulations for controlling and punishing offenders are weak and should be revised as soon as possible. Drivers' mistakes are not always the reason for accidents. In many cases, pedestrian 
create impossible circumstances. Many pedestrian lose their lives because of lack of sidewalks or crossing the road where there are no signs. Pedestrians' education and awareness should be improved as soon as possible. Knowledge and awareness can save pedestrian lives and car losses effectively. This education should be provided and encouraged through the television, newspapers, radio, and posters.

\section{Conflicts of Interest}

The author declares no conflicts of interest regarding the publication of this paper.

\section{References}

[1] World Health Organization (2011) Burden of Disease Project, Burden of Disease 2011 Estimate. http://www.who.int/mediacentre/factsheets/fs310/en/index.html

[2] Aftab News (2016) http://aftabnews.ir/vdciqqazwtlawv2.cbct.html

[3] Reshma, E.K. and Sharif, S.U. (2012) Prioritization of Accident Black Spots Using GIS. International Journal of Emerging Technology and Advanced Engineering, 2, $117-122$.

[4] Fars News (2016) http://www.farsnews.com/newstext.php?nn=13910503000432

[5] Presidency of Islamic Republic of Iran, Management and Planning Organization, Statistical Centerof Iran (2011) Statistical Yearbook. Tehran, Iran.

[6] Department of Transportation and Traffic Organization of Tehran Municipality. http://traffic.tehran.ir/

[7] Kusselson, S.B. (2006) Investigating How Land Sue Patterns Affect Traffic Accident Rates near Frontage Road Cross-Section: A Case Study on Interstate 610 in Houston, Texas. Master Thesis, Graduate College of the Oklahoma State University.

[8] Karacacasu, M., Er, A., Bilgic, S. and Barut, H.B. (2011) Variations in Traffic Accidents on Seasonal, Monthly, Daily and Hourly Basis: Eskisehir Case. Procedia-Social and Behavioral Sciences, 20, 765-775. https://doi.org/10.1016/j.sbspro.2011.08.085

[9] Won-Kyung, L., Hye-Ah, L., Seung-sik, H., Ho, K., Youn-Hee, L., Yun-Chul, H., Eun-Hee, H., and Hyesook, P. (2015) Does Temperature Modify the Effects of Rain and Snow Precipitation on Road Traffic Injuries? Journal of Epidemiology, 25, 544-552.

[10] Dissanayake, D., Aryaija, J. and Wedegama, D.M.P. (2009) Modelling the Effects of Land Use and Temporal Factors on Child Pedestrian Casualties. Accident Analysis and Prevention, 41, 1016-1024. https://doi.org/10.1016/j.aap.2009.06.015

[11] Ben-Akiva, M.E. and Bowman, J.L. (1995) Activity-Based Modelling System for Travel Demand Forecasting. US Department of Transportation and US Environmental Protection Agency, Washington, DC.

http://citeseerx.ist.psu.edu/viewdoc/download?doi=10.1.1.634.7812\&rep=rep1\&type $=$ pdf

[12] Pendar News Agency (2016) https://www.pendaronline.com/

[13] Subramani, T. (2012) Parking Study on Main Corridors in Major Urban Centre. International Journal of Modern Engineering Research, 2, 742-748.

[14] Kim, K.E. and Yamashita, E.Y. (2001) Asleep at the Wheel: Spatial and Temporal Patterns of Fatigue-Related Crashes in Honolulu. Transportation Research Record, 
1779, 46-53. https://doi.org/10.3141/1779-07

[15] Abdalla, I.M., Robert, R., Derek, B. and McGuicagan, D.R.D. (1997) An Investigation into the Relationships between Area Social Characteristics and Road Accident Casualties. Accident Analysis and Prevention, 29, 583-593.

https://doi.org/10.1016/S0001-4575(97)00011-0 